Marquette University

e-Publications@Marquette

2016

\title{
A Full-Dimensional Model of Ozone Forming Reaction: The Absolute Value of the Recombination Rate Coefficient, Its Pressure and Temperature Dependencies
}

Alexander Teplukhin

Marquette University

Dmitri Babikov

Marquette University, dmitri.babikov@marquette.edu

Follow this and additional works at: https://epublications.marquette.edu/chem_fac

Part of the Chemistry Commons

\section{Recommended Citation}

Teplukhin, Alexander and Babikov, Dmitri, "A Full-Dimensional Model of Ozone Forming Reaction: The Absolute Value of the Recombination Rate Coefficient, Its Pressure and Temperature Dependencies" (2016). Chemistry Faculty Research and Publications. 541.

https://epublications.marquette.edu/chem_fac/541 


\title{
A Full-Dimensional Model of Ozone Forming Reaction: The Absolute Value of The Recombination Rate Coefficient, Its Pressure and Temperature Dependencies
}

\author{
Alexander Teplukhin \\ Department of Chemistry, Marquette University, \\ Milwaukee, WI \\ Dmitri Babikov \\ Department of Chemistry, Marquette University, \\ Milwaukee, WI
}

Rigorous calculations of scattering resonances in ozone are carried out for a broad range of rotational excitations. The accurate potential energy surface of Dawes is adopted, and a new efficient method for calculations of rovibrational energies, wave functions and resonance lifetimes is employed (which uses hyper-spherical coordinates, the sequential diagonalization/truncation approach, grid optimization and complex absorbing potential). A detailed analysis is carried out to characterize distributions of resonance energies and lifetimes, their rotational/vibrational content and their positions with respect to the centrifugal barrier. Emphasis is on the

Physical Chemistry Chemical Physics, No. 18 (2016): pg. 19194-19206. DOI. This article is @ Royal Society of Chemistry and permission has been granted for this version to appear in e-Publications@Marquette. Royal Society of Chemistry does not grant permission for this article to be further copied/distributed or hosted elsewhere without the express permission from Royal Society of Chemistry. 
contribution of these resonances to the recombination process that forms ozone. It is found that major contributions come from localized resonances at energies near the top of the barrier. Delocalized resonances at higher energies should also be taken into account, while very narrow resonances at low energies (trapped far behind the centrifugal barrier) should be treated as bound states. The absolute value of the recombination rate coefficient, its pressure and temperature dependencies are obtained using the energytransfer model developed in the earlier work. Good agreement with experimental data is obtained if one follows the suggestion of Troe, who argued that the energy transfer mechanism of recombination is responsible only for $55 \%$ of the recombination rate (with the remaining $45 \%$ coming from the competing chaperon mechanism).

\section{Introduction}

Atmospheric ozone, $\mathrm{O}_{3}$, is formed as a product of recombination reaction of oxygen molecules, $\mathrm{O}_{2}$, with oxygen atoms, $\mathrm{O}$, but the mechanism of this process in not yet entirely understood. In the past, the energy-transfer mechanism, also known as the Lindeman mechanism, was assumed almost exclusively, ${ }^{1,2}$ according to which a metastable ozone molecule is formed at the first step of the process, and is stabilized at the second step by collision with an atom or a molecule of the bath gas (e.g., $\mathrm{Ar}, \mathrm{N}_{2}$ ):

$$
\begin{aligned}
& \mathrm{O}_{2}+\mathrm{O} \rightarrow \mathrm{O}_{3}^{*},(1) \\
& \mathrm{O}_{3} *+\mathrm{M} \rightarrow \mathrm{O}_{3}+\mathrm{M}^{*} .(2)
\end{aligned}
$$

Here, the role of $M$ is to quench the metastable intermediate $\mathrm{O}_{3} *$, producing a stable ozone molecule. However, a more recent analysis of the experimental data ${ }^{3}$ indicates that this may not be the only and, in fact, not necessarily the dominant mechanism of ozone formation. It looks like simultaneously with the energy-transfer mechanism described above, the so-called chaperon mechanism, also known as the radical-complex mechanism, may produce ozone via:

$$
\mathrm{O}+\mathrm{M} \rightarrow \mathrm{OM}^{*}
$$




\section{$\mathrm{O}_{2}+\mathrm{OM}^{*} \rightarrow \mathrm{O}_{3}+\mathrm{M}^{*} .(4)$}

Here the roles of $M$ are to (non-covalently) bind the oxygen atom, exchange it with $\mathrm{O}_{2}$ and, finally, carry away the excess energy, leaving a stable ozone molecule behind. Experimentally it is not straightforward to tell the difference between these two mechanisms since, under the steady state conditions, each of them leads to the third-order kinetics overall, first order in each [O], $\left[\mathrm{O}_{2}\right]$ and $[\mathrm{M}]$. But, based on the analysis of the temperature dependence of the recombination rate coefficient (in a broad range) Troe $^{3}$ was able to determine contributions of each mechanism. For example, at room temperature and atmospheric pressure, in the air bath, the contribution of energy-transfer is close to $40 \%$, while the contribution of the chaperon mechanism is close to $60 \%$ of the total recombination rate. At low temperatures chaperon dominates, while energy-transfer dominates at high temperatures. ${ }^{3}$

Further justification of this observation should come, ideally, from the theory side, but, sadly, neither of these two mechanisms is readily amenable to accurate theoretical treatment, even with the simplest $M$, such as an Ar atom. First of all, the metastable $\mathrm{O}_{3} *$ in reaction (1) and the complex $\mathrm{OM}^{*}$ in reaction (3) represent scattering resonances, characterized by strong ro-vibrational excitation above dissociation threshold and finite lifetimes. Their properties have to be determined using quantum mechanics, which is computationally demanding, particularly in the case of triatomic $\mathrm{O}_{3} *$ where a deep covalent well supports $\sim 280$ vibrational bound states (below dissociation threshold), and the rotational excitation reaches $\mathrm{J} \sim 90$. Moreover, calculations for rotational and vibrational quenching of $\mathrm{O}_{3} *$ in the process (2), using an accurate quantum method for inelastic scattering, are far behind the reach of theorists today. Similarly, the quantum reactive scattering calculations for the process (4) would be close to impossible, and have never been attempted.

It is much easier to set up the classical trajectory simulations for ozone formation, and this has been done for both energy-transfer and chaperon mechanisms, ${ }^{4,5}$ but reliability of those results should not be overemphasized, since application of classical trajectories for the description of quantum resonances is rather controversial, as well as their validity for the description of inelastic scattering of $\mathrm{O}_{3}$, where 
quantum symmetry and zero-point energy are important. Unavoidably, theorists had to stay within the quantum framework, trying various kinds of approximations to ease calculations. Those efforts are reviewed next.

The energy-transfer mechanisms (1) and (2) received most attention, because they are believed to be responsible for anomalous mass-independent fractionation of oxygen isotopes produced by the recombination process. ${ }^{6-8}$ The first systematic calculation of energies and lifetimes of scattering resonances $\mathrm{O}_{3} *$ in reaction (1) was carried out by Babikov et al.9,10 Those data helped us to understand the origin of the anomalous isotope effect in ozone, ${ }^{10,11}$ but were not sufficient for quantitative treatment of recombination kinetics, since they were obtained for a non-rotating ozone molecule only, $\mathrm{J}=0$. At about the same time, Charlo and Clary proposed a dimensionally-reduced model of energy-transfer in ozone ${ }^{12,13}$ where, in order to reduce the number of vibrational degrees of freedom, they fixed the bending angle in $\mathrm{O}_{3}$ (allowing only the stretching motion of two bonds) and employed sudden approximation for $\mathrm{O}_{3} *+\mathrm{Ar}$ collision (which also restricts consideration to a non-rotating ozone molecule, $\mathrm{J}=0$ only). They were the first to make quantitative predictions of the recombination kinetics for the energy-transfer mechanism of ozone formation based on quantum calculations, but some of their results seem to be controversial. For example, their temperature dependence of the recombination rate coefficient was positive, ${ }^{13}$ while it is negative in the experiment. ${ }^{3}$ Also, it is not entirely clear how they compensated for the reduced number of states in a model where all bending states of $\mathrm{O}_{3}$ were missing. ${ }^{12}$

$\mathrm{Xi}$ and Bowman ${ }^{14}$ improved upon this last point, using a method very similar to that of Clary, but with all vibrational degrees of freedom included. In order to make their calculations affordable, they only considered a minimal number of representative collision geometries for $\mathrm{O}_{3} *+\mathrm{Ar}$ encounter. Their results contributed to the interpretation of the isotope effect at the qualitative level, ${ }^{14}$ but the absolute value of the recombination rate coefficient was not computed. A bit later, Ivanov and Schinke ${ }^{15}$ carried out calculations similar to those of Bowman, but with all the collision geometries sampled appropriately, and all the partial waves included for convergence. However, their focus was on state-to-state transitions between the 
bound vibrational states (below dissociation threshold) rather than on scattering resonances. So, the recombination process was not studied. Note that neither Clary, ${ }^{13}$ nor Bowman ${ }^{14}$ or Schinke ${ }^{15}$ computed lifetimes of $\mathrm{O}_{3} *$ resonances. All these calculations employed the sudden collision approximation for $\mathrm{O}_{3}{ }^{*}+\mathrm{Ar}$ collision and were restricted to the $\mathrm{J}=0$ case only (non-rotating $\mathrm{O}_{3} *$ ).

Resonance lifetimes for rotationally excited ozone molecules were computed for the first time by Grebenshchikov and Schinke, ${ }^{16}$ for the range $\mathrm{J} \leq 40$ and $\mathrm{K}_{\mathrm{a}} \leq 10$, but without any treatment of the stabilization step (2), just using a strong-collision assumption for quenching of the metastable $\mathrm{O}_{3} *$ by Ar. They mentioned that this approach allows obtaining reasonable temperature and pressure dependencies of the recombination rate coefficient, but did not present those data, and did not report the absolute value of the coefficient (focusing on the isotope effect, characterized by the ratio of rate coefficients for different isotopomers).

More recently, we developed a mixed quantum/classical method for the description of collisional energy-transfer ${ }^{17,18}$ and applied it to the energy-transfer mechanism of ozone recombination. ${ }^{19-21}$ This approach overcomes many difficulties: sudden collision approximation is avoided and the dynamics of $\mathrm{O}_{3} *+$ Ar scattering is treated in a time-dependent manner, classically; rotational motion of $\mathrm{O}_{3} *$ is incorporated, also classically, and the rotational quenching of $\mathrm{O}_{3} *$ in collisions with $M$ is computed; vibrational motion of ozone is treated quantum mechanically, which describes zero-point energy and symmetry of vibrational modes; scattering resonances are incorporated, including accurate calculations of their lifetimes. ${ }^{17,21}$ Using this approach, we carried out rather massive calculations of formation, decay, stabilization and dissociation of many individual rovibrational resonances in the dimensionally-reduced model of $\mathrm{O}_{3}$ * where, similar to Clary, we neglected excitation of the bending mode of ozone. ${ }^{17}$ Those calculations gave detailed insight into the recombination process ${ }^{20,21}$ and, also, allowed us to derive a simple analytical formula for the description of energy-transfer in ozone. ${ }^{22}$

The only serious limitation of our previous work was the dimensionally reduced approximation for $\mathrm{O}_{3} *$. Indeed, if excitation of the bending mode is not allowed, then the total number of vibrational 
states in $\mathrm{O}_{3} *$ is smaller (roughly, by a factor of six) compared to the real ozone molecule, which translates into a lowered recombination rate. ${ }^{22}$ In principle, the experimental recombination rate can be recovered by an ad hoc adjustment (e.g., based on statistical argument of the density of states, as we did in ref. 21 ), but it is certainly desirable to develop a complete treatment of ozone recombination, including all degrees of freedom.

In this paper we do exactly that. Using a method that employs hyper-spherical coordinates, the sequential diagonalization-truncation technique, and complex absorbing potential ${ }^{23}$ we compute energies and lifetimes of scattering resonances in a full-dimensional model of ozone (including its bending motion) for a broad range of rotational excitations, up to $\mathrm{J}=64, \mathrm{~K}_{\mathrm{a}} \leq \mathrm{J}$. This information, together with the analytic formula for collisional energy-transfer and collision-induced dissociation derived earlier, ${ }^{22}$ permits building a more complete version of the energy-transfer mechanism of ozone recombination. When complemented by contribution of the chaperon mechanism (as suggested by Troe $^{3}$ ) our results agree well with experimental data, including the absolute value of the recombination rate coefficient, its temperature and pressure dependencies.

This study is carried out only for the most abundant isotopically unsubstituted $\mathrm{O}_{3}$, composed of three ${ }^{16} \mathrm{O}$ atoms, since majority of experimental kinetics data are available for this isotopic combination, and since the rates for a variety of isotopically-substituted ozone species (e.g., ${ }^{16} \mathrm{O}^{16} \mathrm{O}^{18} \mathrm{O}$ ) are always given relative to the rate of ${ }^{16} \mathrm{O}^{16} \mathrm{O}^{16} \mathrm{O}$ formation. Similar studies for several other isotopic combinations will be done in the next step.

\section{Theoretical framework}

\section{II-A. Reaction mechanism}

Kinetics of the processes ( 1 and 2 ) is described within the micro-canonical framework, where different scattering resonances of $\mathrm{O}_{3} *$ are treated as different chemical species. ${ }^{10,12,16}$ For each scattering resonance $\mathrm{O}^{(i)}{ }_{3}$ at energy $\mathrm{E}_{i}$ the processes affecting its population 
$\left[\mathrm{O}^{(i)}{ }_{3}\right]$ are considered and the corresponding rate constants are introduced. Those are:

(i) Formation of $\mathrm{O}^{(\mathrm{i})}{ }_{3}$ from $\mathrm{O}_{2}+\mathrm{O}$ characterized by the secondorder rate coefficient $\mathrm{k}^{\text {form }_{\mathrm{i}} \text { : }}$

$$
\mathrm{O}_{2}+\mathrm{O} \rightarrow \mathrm{O}^{(\mathrm{i})}{ }_{3} ;(5)
$$

(ii) Spontaneous unimolecular decay of $\mathrm{O}^{(\mathrm{i})}{ }_{3}$ onto $\mathrm{O}_{2}+\mathrm{O}$ characterized by the first-order rate coefficient $k^{\text {dec }_{i} \text { : }}$

$$
\mathrm{O}^{(\mathrm{i})} \mathrm{O}_{3} \rightarrow \mathrm{O}_{2}+\mathrm{O} \text {; (6) }
$$

(iii) Stabilization of $\mathrm{O}^{(\mathrm{i})}{ }_{3}$ by collision with a bath gas atom characterized by the second-order rate coefficient $\mathrm{k}^{\text {stab }_{i}}$ :

$$
\mathrm{O}^{(\mathrm{i})}{ }_{3}+\mathrm{M} \rightarrow \mathrm{O}_{3}+\mathrm{M}^{*} \text {; (7) }
$$

(iv) Collision-induced dissociation $\mathrm{O}^{(\mathrm{i})}{ }_{3}$ onto $\mathrm{O}_{2}+\mathrm{O}$ characterized by the second-order rate coefficient $\mathbf{k}^{\text {diss }}$ :

$$
\mathrm{O}^{(\mathrm{i})}{ }_{3}+\mathrm{M}^{*} \rightarrow \mathrm{O}_{2}+\mathrm{O}+\mathrm{M} \text {. (8) }
$$

The width $\Gamma_{i}$ of quantum scattering resonance $\mathrm{O}^{(i)}{ }_{3}$, computed as explained in Section II-B, gives us directly the value of its decay rate, $k^{\text {dec }_{i}}=\Gamma_{i}$. The coefficients $k^{\text {form }_{i}}$ and $k^{\text {dec }}$ for each scattering resonance are related to each other through a micro-canonical equilibrium constant: $k^{\text {form }}{ }_{i}=1 / 2 k^{\text {dec }} K_{\text {eq }}\left(E_{i}\right)$. Here the factor of $1 / 2$ reflects the fact that while in a symmetric ${ }^{48} \mathrm{O}_{3}$ there are two equivalent channels for the decay of resonances, the formation rate coefficient $k^{\text {form }} m_{i}$ is per one entrance channel, simply because $\mathrm{O}$ hits only one side of $\mathrm{O}_{2}$ in a given encounter. The equilibrium constant $\mathrm{K}_{\mathrm{eq}}\left(\mathrm{E}_{\mathrm{i}}\right)$ for each scattering resonance is computed statistically using a known formula, ${ }^{20}$ and taking into account a symmetry number of $1 / 2$ for the partition function of symmetric reagent ${ }^{32} \mathrm{O}_{2}$ (in denominator), where only odd rotational states are allowed. The values of $\mathrm{k}^{\text {stab }_{i}}$ and $\mathrm{k}^{\text {diss }_{i}}$ are computed based on the results of the mixed quantum/classical simulations for $\mathrm{O}^{(\mathrm{i})}{ }_{3}+\mathrm{Ar}$ collision dynamics, ${ }^{22}$ as explained in Section II-C.

Assuming steady-state conditions for the concentration of each state $\mathrm{O}^{(\mathrm{i})}{ }_{3}$ allows deriving analytic expression for the third-order recombination rate coefficient of the overall recombination reaction: 


$$
\kappa=\sum_{i} \frac{\frac{1}{2} \Gamma_{i} K_{\text {eq }}\left(E_{i}\right)}{\Gamma_{i}+\left(k_{j}^{\text {stab }}+k_{i}^{\text {diss }}\right)[\mathrm{M}]} k_{j}^{\text {stab }} \text {, }
$$

where the sum is over all scattering resonances $\mathrm{O}^{(i)}$. As explained above, this recombination rate coefficient is per one formation channel.

Several processes, less important than (5-8), are neglected in our treatment of kinetics. Namely, we do not include the possibility of back excitations, $\mathrm{O}_{3}+\mathrm{Ar} \rightarrow \mathrm{O}^{(\mathrm{i})}{ }_{3}+\mathrm{Ar}$, assuming that concentration of the formed ozone $\left[\mathrm{O}_{3}\right]$ is small. We also neglect the collision-induced transitions between different scattering resonances, $\mathrm{O}^{(\mathrm{i})}{ }_{3}+\mathrm{Ar} \rightarrow \mathrm{O}^{(\mathrm{j})_{3}}+$ $\mathrm{Ar}$, assuming that their populations are entirely determined by equilibrium with reagents $\mathrm{O}_{2}+0$. These assumptions are reasonable at low and moderate pressures of the bath gas.

\section{II-B. Energies, widths and wave functions of resonances}

A numerical method used to determine the properties of scattering resonances is reported in detail elsewhere. ${ }^{23}$ Here we give only a brief summary. Three vibrational degrees of freedom in $\mathrm{O}_{3}$ are described using adiabatically-adjusting principal-axis hyper-spherical coordinates $\rho, \theta$ and $\varphi .{ }^{24}$ For low-amplitude vibrations near the equilibrium geometry of ozone ( $\rho_{\text {eq }}=4.048$ Bohr) the motion along the hyper-radius $\rho$ corresponds to the breathing mode in $\mathrm{O}_{3}$. For typical levels of rotational excitation the top of the centrifugal barrier occurs near $\rho^{\dagger} \approx 5.4$ Bohr. Wave functions of scattering resonances trapped behind the centrifugal barrier are localized mostly in the range $\rho<\rho^{\dagger}$. In the asymptotic (channel) region the motion along $\rho$ describes dissociation of $\mathrm{O}_{3}$ onto $\mathrm{O}_{2}+\mathrm{O}$. Complex absorbing potential in the form suggested by Balint-Kurti ${ }^{25}$ is placed in the range $10<\rho<$ 15 Bohr in order to absorb the tails of resonant wave functions. Hyperangles $\theta$ and $\varphi$ describe bending and asymmetric-stretching motions near the equilibrium point. The symmetry of the vibrational wave function is determined by reflection through $\varphi=0$. For symmetric ${ }^{48} \mathrm{O}_{3}$ vibrational wave functions are either symmetric $\left(A_{1}\right)$ or antisymmetric $\left(A_{2}\right)$. 
The potential energy surface of Dawes ${ }^{26}$ was used in our calculations. Compared to the older surfaces of ozone, ${ }^{9-11,27,28}$ this new surface has slightly better dissociation energy (compared to the most advanced experimental data), and slightly different behavior along the minimum energy path for dissociation (a flat "shoulder", rather than a small submerged "reef"), which gives better agreement with experiment for the atom-exchange process. ${ }^{29-33}$ Other than that, the older and new surfaces are very similar, and exhibit very similar densities of states near threshold. ${ }^{34}$

We found that it is impossible to come up with a 3D-grid that covers uniformly and efficiently the entire configuration space of the problem. ${ }^{35}$ So, a straightforward diagonalization of the Hamiltonian matrix using a 3D-grid was found to be prohibitively expensive computationally. In order to make calculations feasible we employed the sequential diagonalization-truncation approach of Bačić and Light, ${ }^{36,37}$ adapted to the hyper-spherical coordinates. Namely, for each value of $\rho$ on the grid, we determined solutions of a twodimensional problem in $\theta$ and $\varphi$, and used those as locally optimal basis sets for efficient representation of the global 3D wave function (of the given symmetry, separately for $A_{1}$ and $A_{2}$ ). The grid along $\rho$ was also optimized to reflect the shape of the potential energy surface, using a method based on the local value of de Broglie wave length. 38,39 We found that this combined FBR/DVR approach is very efficient. ${ }^{23}$ Complex eigenvalues $\mathrm{E}-\mathrm{i} \Gamma / 2$ and wave functions of the reduced matrix were computed using the ScaLAPACK package. ${ }^{40}$ Instead of the scattering approach (coupled-channel, often used in conjunction with hyper-spherical coordinates), we solved a 3D-eigenvalue problem, with complex absorbing potential introduced in the asymptotic range of the PES. All details of our method will be given in the forthcoming methodological paper. ${ }^{23}$

Similar to the previous work by Grebenshchikov and Schinke, ${ }^{16}$ we adopted the centrifugal-sudden approximation, known also as symmetric-top rotor approximation, or K-conserving approximation (where $\mathrm{K}$ is projection of total angular momentum $\mathrm{J}$ onto the first principal axis of inertia, i.e. $\mathrm{K}=\mathrm{K}_{\mathrm{a}}$, for each instantaneous molecular configuration). This is the only approximation used here. It involves neglecting the Coriolis coupling term in the Hamiltonian operator, but also neglecting the asymmetric-top term $(A-B) / 2$ in the rotational 
potential. As was emphasized by Parker and Pack, ${ }^{41}$ these two simplifications constitute one single approximation, and have to be made simultaneously in order to decouple rotational and vibrational degrees of freedom. Thus, our calculations were done independently for different values of $\mathrm{K}_{\mathrm{a}} \leq \mathrm{J}$. Vibrational wave functions of both symmetries, $A_{1}$ and $A_{2}$, were retained, since they would contribute to solutions of different parities in the exact fully-coupled approach, except the case of $K_{a}=0$, when only $A_{2}$ solutions were kept for $\mathrm{J}=0$, only $A_{1}$ solutions for $\mathrm{J}=1$, then, again, only $A_{2}$ solutions for $\mathrm{J}=2$, and so on (see for example, ref. 34 ). Note that states of symmetry $E$ are not physically allowed for ${ }^{16} \mathrm{O}^{16} \mathrm{O}^{16} \mathrm{O}$, since in this case the wave function must be symmetric with respect to permutation of any two oxygen atoms. Thus, E-symmetry states were excluded from calculations, by restricting the range of hyper-angle $\varphi$ to one well only, $120^{\circ} \leq \varphi \leq 240^{\circ}$.

Typically, the Coriolis coupling is non-negligible in the "floppy" molecules only. In the "stiff" molecules, such as ozone, neglecting this coupling term is well justified and has been done in the past by other authors. ${ }^{16}$ If needed, the action of this term onto wave function can be rigorously evaluated. ${ }^{41,42}$ In the future we plan testing the effect of Coriolis coupling by including it, at least, for the states with small $\mathrm{K}$ values (e.g., $K<7$ ), while neglecting it for larger $K$.

\section{II-C. Stabilization and dissociation rates}

Rate coefficients for stabilization $\mathrm{k}^{\mathrm{stab}_{i}}$ of scattering resonances $\mathrm{O}^{(\mathrm{i})}{ }_{3}$ are obtained from the corresponding cross sections:

$$
k_{i}^{\text {sab }}=\sigma_{i}^{\text {sab }}\left(\frac{8 k T}{\pi \mu}\right)^{1 / 2} .
$$

where $\mu$ is $\mathrm{O}_{3}+A r$ reduced mass, and similar for the $\mathrm{k}^{\text {diss }}{ }_{\mathrm{i}}-$ rate coefficient for the dissociation of $\mathrm{O}^{(\mathrm{i})}$. In our previous work we generated several sets of data that are used here to compute $\sigma^{s t a b}{ }_{i}\left(E_{i}\right)$ and $\sigma^{\text {diss }}{ }_{i}\left(E_{i}\right)$.

First of all, one can use the analytical formula obtained in ref. $\underline{22}$ to represent on average a large amount of data obtained for 
stabilization of various scattering resonances in four different isotopomers of ozone. In that case, for a resonance at energy $E$ above dissociation threshold, the value of stabilization cross section is given by

$$
\sigma^{\mathrm{sab}}(E)=\sigma_{0}^{\mathrm{sab}}\left(\exp \left\{-\frac{E}{\gamma}\right\}+c \exp \left\{-\frac{E}{d}\right\}\right)
$$

The values of parameters $\sigma^{\text {stab }}{ }_{0}, \mathrm{C}, \mathrm{Y}$ and $\mathrm{d}$ for this model can be found in Table 2 of ref. 22. A slightly different fit ${ }^{22}$ of the same data uses two variables, $\mathrm{E}$ and $\mathrm{E}^{\mathrm{vib}}$ :

$$
\begin{aligned}
\sigma^{\mathrm{sabb}}\left(E, E^{\mathrm{vib}}\right)= & \sigma_{0}^{\mathrm{sab}}\left(\exp \left\{-\frac{E}{\gamma}\right\}+c \exp \left\{-\frac{E}{d}\right\}\right) \\
& \times\left(1-\frac{A_{\mathrm{v}}}{1+\left(E^{\mathrm{vib}} / k T\right)^{2}}\right) .
\end{aligned}
$$

Here $E^{\text {vib }}$ is vibrational energy of the resonance, which is total energy $E$ less rotational energy, and $A_{v}$ is one more fitting parameter. ${ }^{22}$ This formula reflects observation that the stabilization process is influenced by the balance between vibrational and rotational content of the resonance. Namely, stabilization cross sections are larger for those states where rotational excitation is larger, because rotational energy is exchanged more readily in a typical $\mathrm{O}^{(\mathrm{i})}{ }_{3}+\mathrm{Ar}$ collision. Eqn (11) and (12) will be referred to as versions a and b of Stabilization Model 1, or SM1a and SM1b. The average dissociation cross section of a resonance at energy $\mathrm{E}$ is computed using the following formula: ${ }^{20}$

$$
\sigma^{\text {diss }}(E)=\sigma_{0}^{\text {diss }}\left(1+\tanh \left\{\frac{\left(E-E_{0}^{\text {diss }}\right)}{\gamma^{\text {diss }}}\right\}\right),
$$

and a set of parameters $\sigma^{\text {diss }}{ }_{0}, E_{0}$ diss and $Y^{\text {diss }}$ that can be found in ref. $\underline{22}$.

Alternatively, one can use the results of ref. 20, where we determined the energy-transfer functions $\bar{\sigma}_{i}^{\text {tran }}(\Delta \mathrm{E})$ for several 
individual resonances (ten representative states) and fitted each separately by a double-exponential analytic model (see Fig. 7 in ref. $\underline{20}$ for examples of such energy-transfer functions and Table 4 in ref. 20 for the values of fitting parameters). Such energy-transfer functions $\bar{\sigma}^{\operatorname{tran}}(\Delta \mathrm{E})$, measured in the units of $\mathrm{a}_{0}{ }^{2} / \mathrm{cm}^{-1}$, can be analytically integrated through the range $[-\infty ;-E]$ in order to obtain stabilization cross sections, in the units of $\mathrm{a}_{0}{ }^{2}$, for a resonance at energy $E$ above the dissociation threshold:

$$
\sigma^{\mathrm{sab}}(E)=\int_{-\infty}^{-E} \bar{\sigma}^{\mathrm{iran}}\left(E^{\prime}\right) \mathrm{d} E^{\prime}
$$

Employing the data for ten different resonances studied in ref. 20 we introduce some range of typical values for the stabilization cross section $\sigma^{\text {stab }}(E)$, rather than one definite number. This approach is further referred to as Stabilization Model 2, or SM2.

So, in this work the stabilization cross sections are not computed for each individual resonance (e.g., by solving the $\mathrm{O}^{(\mathrm{i})}{ }_{3}+\mathrm{Ar}$ collision problem numerically), rather they are obtained approximately, by substituting resonance energy $E$ (and $E^{\text {vib }}$ for SM1b) computed in Section II-B, into analytic expressions of eqn (11)-(13). Note, however, that parameters of eqn (11)-(13) were determined in the earlier work, ${ }^{22}$ based on the mixed quantum/classical modeling of $\mathrm{O}^{(\mathrm{i})}{ }_{3}$ + Ar collisions.

\section{Results and discussion}

\section{III-A. Properties of resonances}

In this section we analyze the collective properties of resonances in $\mathrm{O}_{3} *$ with emphasis on their contribution to the overall process of recombination, rather than the properties of individual resonances, simply because hundreds of resonances are involved. Thus, the histogram of Fig. 1a represents contribution of resonances to

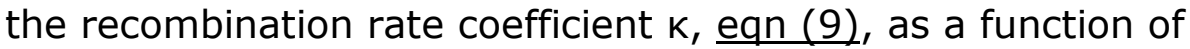
resonance energy above the dissociation threshold of $\mathrm{O}_{3}$ (which includes ro-vibrational zero-point energy of the $\mathrm{O}_{2}$ product in the asymptotic/channel range). We see that the maximum of this 
distribution is close to $E_{i}=100 \mathrm{~cm}^{-1}$. Lower energy resonances (closer to threshold) contribute less. The tail of distribution extends up to $\mathrm{E}_{\mathrm{i}}=$ $800 \mathrm{~cm}^{-1}$. Similarly, the histogram of Fig. $1 \mathrm{~b}$ represents the distribution of resonance widths, and we see that major contributions to the recombination rate coefficient $\mathrm{k}$ come from resonances characterized by widths in the range $10^{-2}<\Gamma_{\mathrm{i}}<10 \mathrm{~cm}^{-1}$, with maximum of the distribution around $\Gamma_{\mathrm{i}} \approx 1 \mathrm{~cm}^{-1}$.

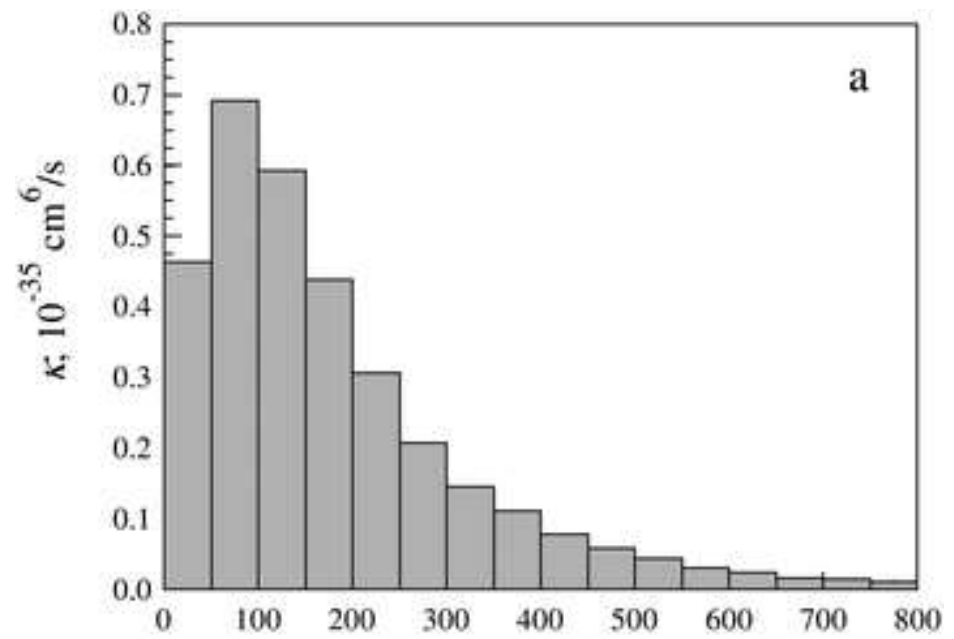

$E, \mathrm{~cm}^{-1}$

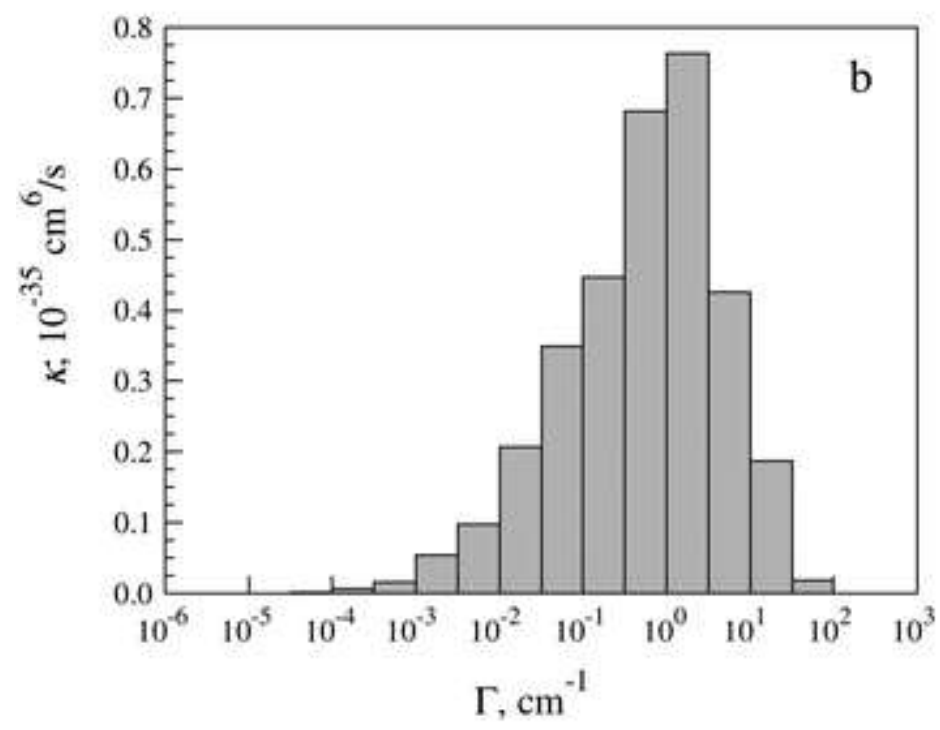

Fig. 1 Contributions of scattering resonances to the recombination rate coefficient $k$ as a function of (a) resonance energy $E_{i}$ above the dissociation threshold; and (b) resonance widths $\Gamma_{\mathrm{i}}$.

Fig. 2 allows seeing a correlation between energy $E_{i}$ and width $\Gamma_{i}$ of resonances, again, with the focus on those states that are important

Physical Chemistry Chemical Physics, No. 18 (2016): pg. 19194-19206. DOI. This article is @ Royal Society of Chemistry and permission has been granted for this version to appear in e-Publications@Marquette. Royal Society of Chemistry does not grant permission for this article to be further copied/distributed or hosted elsewhere without the express permission from Royal Society of Chemistry. 
for the recombination process. Color in Fig. 2 indicates contribution to the recombination rate coefficient $\mathrm{k}$. Fig. $1 \mathrm{a}$ and $\mathrm{b}$ are projections of the data in Fig. 2 onto horizontal and vertical axes, $E$ and $\Gamma$, respectively. Distribution of Fig. 2 is not particularly broad: the most intense part of it spans only the $300 \mathrm{~cm}^{-1}$ range of resonance energies and three orders of magnitude range of resonance widths.

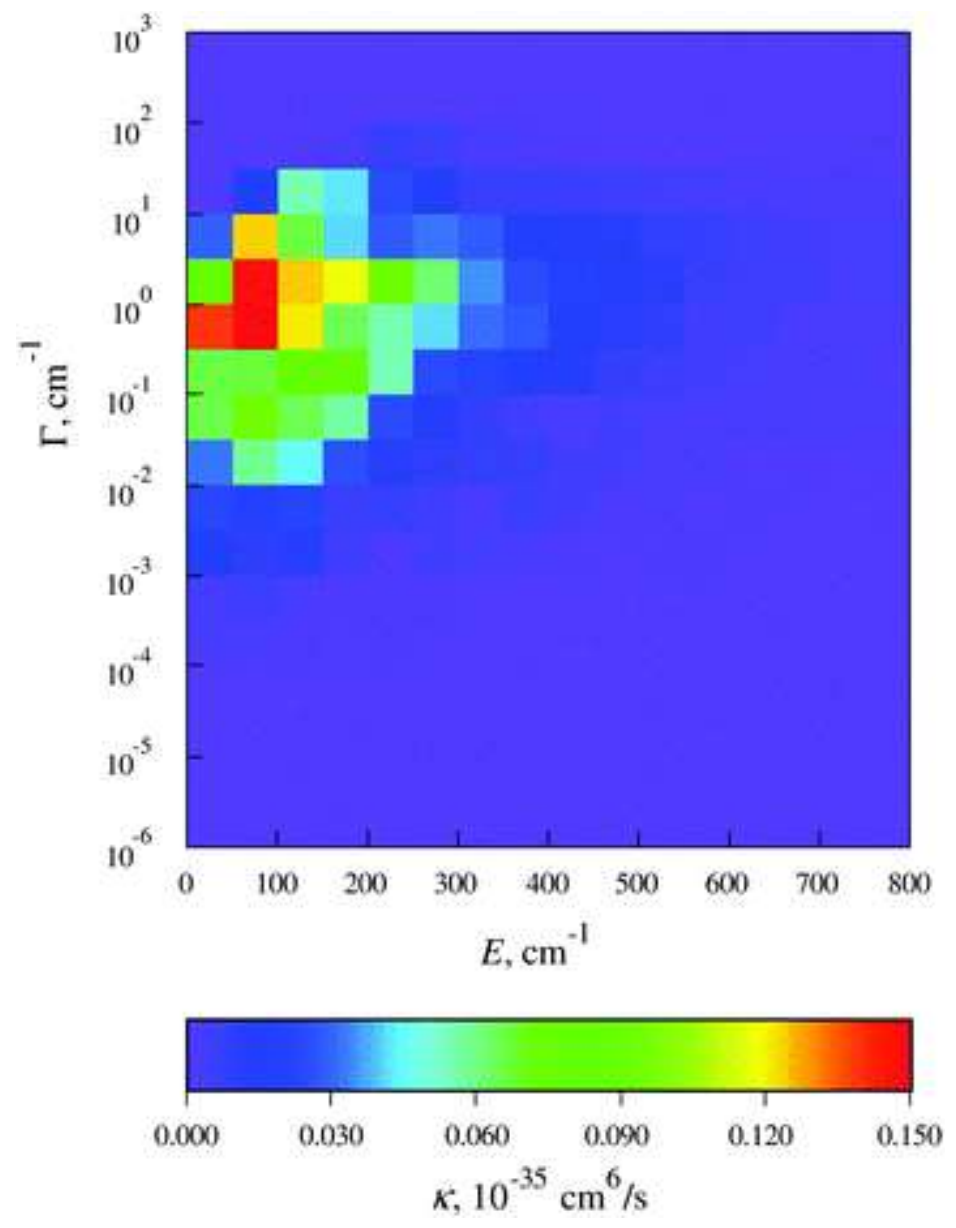

Fig. 2 Contributions of scattering resonances to the recombination rate coefficient $k$ as a function of both resonance energy $E_{i}$ and width $\Gamma_{i}$. Projections of this $2 D$ distribution onto horizontal and vertical axes gives Fig. 1a and b, respectively.

Fig. 3 represents contribution of different rotational excitations to the recombination process. Color indicates the value of $\mathrm{k}\left(\mathrm{J}, \mathrm{K}_{\mathrm{a}}\right)$ obtained using eqn (9) where summation was carried out over the vibrational states only, within each rotational state characterized by J and $\mathrm{K}_{\mathrm{a}}$. The distribution in Fig. 3 indicates that the most important 
contributions to the recombination process come from rotational excitations in the range $8<\mathrm{J}<38$ and $K<7$, which is close to the range studied by Grebenshchikov and Schinke. ${ }^{16}$ Since many vibrational states are included, the distribution of Fig. 3 is rather shapeless. Two (not particularly well pronounced) maxima barely seen in Fig. 3 correspond to the states of symmetries $A_{1}$ and $A_{2}$ that exhibit slightly different properties. Note that low rotational excitations, say $\mathrm{J}$ $<5$, make only a negligible contribution to the process. This means that any prediction or analysis based on calculations for $\mathrm{J}=0$ only (non-rotating $\mathrm{O}_{3}$ ) is likely to be inaccurate, or may even be qualitatively wrong. Importantly, calculations with J > 45 are not really needed.

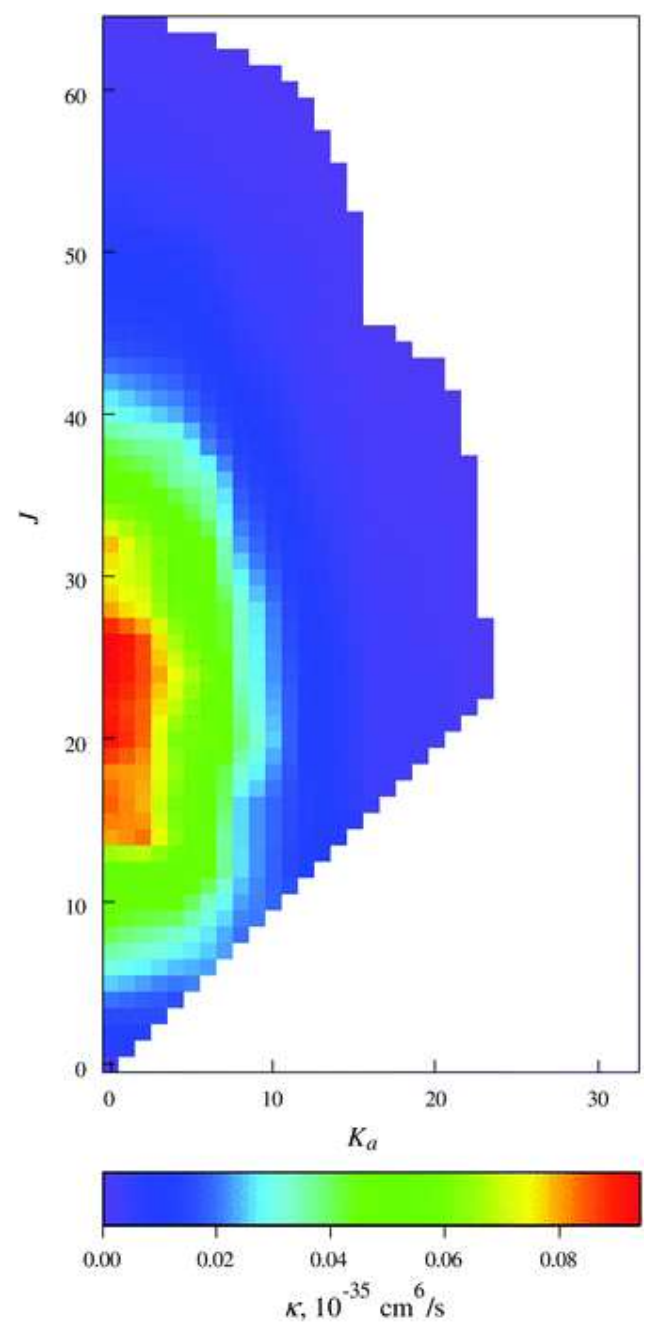

Fig. 3 Contributions of scattering resonances to the recombination rate coefficient $k$ as a function of rotational excitation $(\mathrm{J}, \mathrm{K})$. Step size $\Delta \mathrm{J}=\Delta \mathrm{K}=2$ was used in the

Physical Chemistry Chemical Physics, No. 18 (2016): pg. 19194-19206. DOI. This article is @ Royal Society of Chemistry and permission has been granted for this version to appear in e-Publications@Marquette. Royal Society of Chemistry does not grant permission for this article to be further copied/distributed or hosted elsewhere without the express permission from Royal Society of Chemistry. 
range $12 \leq \mathrm{J} \leq 36$ and $\mathrm{K} \leq 4$; it was doubled in the range $\mathrm{J} \leq 44$ and $\mathrm{K} \leq 16$ and doubled again in the range $\mathrm{J} \leq 64$ and $\mathrm{K} \leq 32$. Contributions of all other rotational excitations were linearly interpolated between the computed points.

Vibrational content of resonances in $\mathrm{O}_{3} *$ can be analyzed by determining the state number of the resonance within progression of vibrational states computed for given values of $\mathrm{J}$ and $\mathrm{K}_{\mathrm{a}}$, and by matching energy of the corresponding state in the spectrum of nonrotating ozone (can be thought of as vibrational energy of the resonance). The distribution of state numbers is given separately for symmetries $A_{1}$ and $A_{2}$ in two frames of Fig. 4 . We see that most significant contributions to the recombination process come from the state number 120 -to- 155 of symmetry $A_{1}$, and the state number 90 to-115 of symmetry $A_{2}$. Note that a non-rotating ozone molecule has 163 states of symmetry $A_{1}$, and 125 states of symmetry $A_{2}$ (using the PES of Dawes ${ }^{26}$ ). This means that upper vibrational states, closest to the dissociation threshold, are more important for the recombination process. In Fig. 5 we gave a distribution of energies of these states, relative to the dissociation threshold, for both symmetries combined. This histogram shows that dominant contributions to recombination come from vibrational states in the range $600 \mathrm{~cm}^{-1}$ below the dissociation threshold. Such states contain 10 to 13 quanta of vibrational excitation distributed between three modes. Typical examples include 6 to 8 quanta of bending and/or asymmetric stretching, and 4 to 5 quanta of symmetric stretching. Some states have only two modes excited (e.g., 11 quanta of $\theta$ and 1 quanta of $\varphi$ ), or even a single mode (e.g., 12 quanta of bending). Properties of these vibrational states, including a detailed analysis of their wave functions, are reported elsewhere. ${ }^{23}$ 
NOT THE PUBLISHED VERSION; this is the author's final, peer-reviewed manuscript. The published version may be accessed by following the link in the citation at the bottom of the page.
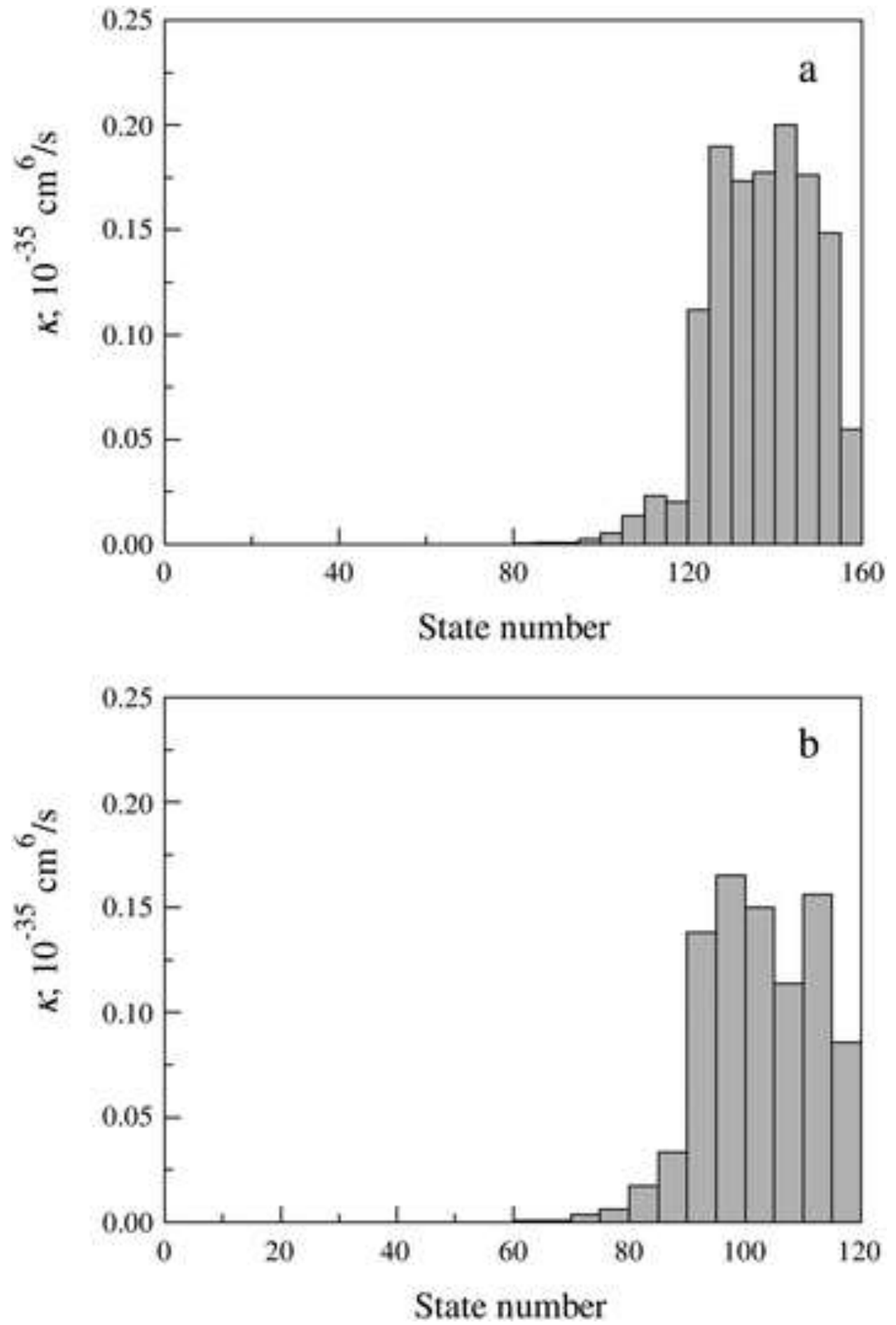

Fig. 4 Contributions of scattering resonances to the recombination rate coefficient $k$ as a function of the state number in a progression of (a) symmetric and (b) antisymmetric vibrational states. Only resonances localized in the well are included.

Physical Chemistry Chemical Physics, No. 18 (2016): pg. 19194-19206. DOI. This article is @ Royal Society of Chemistry and permission has been granted for this version to appear in e-Publications@Marquette. Royal Society of Chemistry does not grant permission for this article to be further copied/distributed or hosted elsewhere without the express permission from Royal Society of Chemistry. 


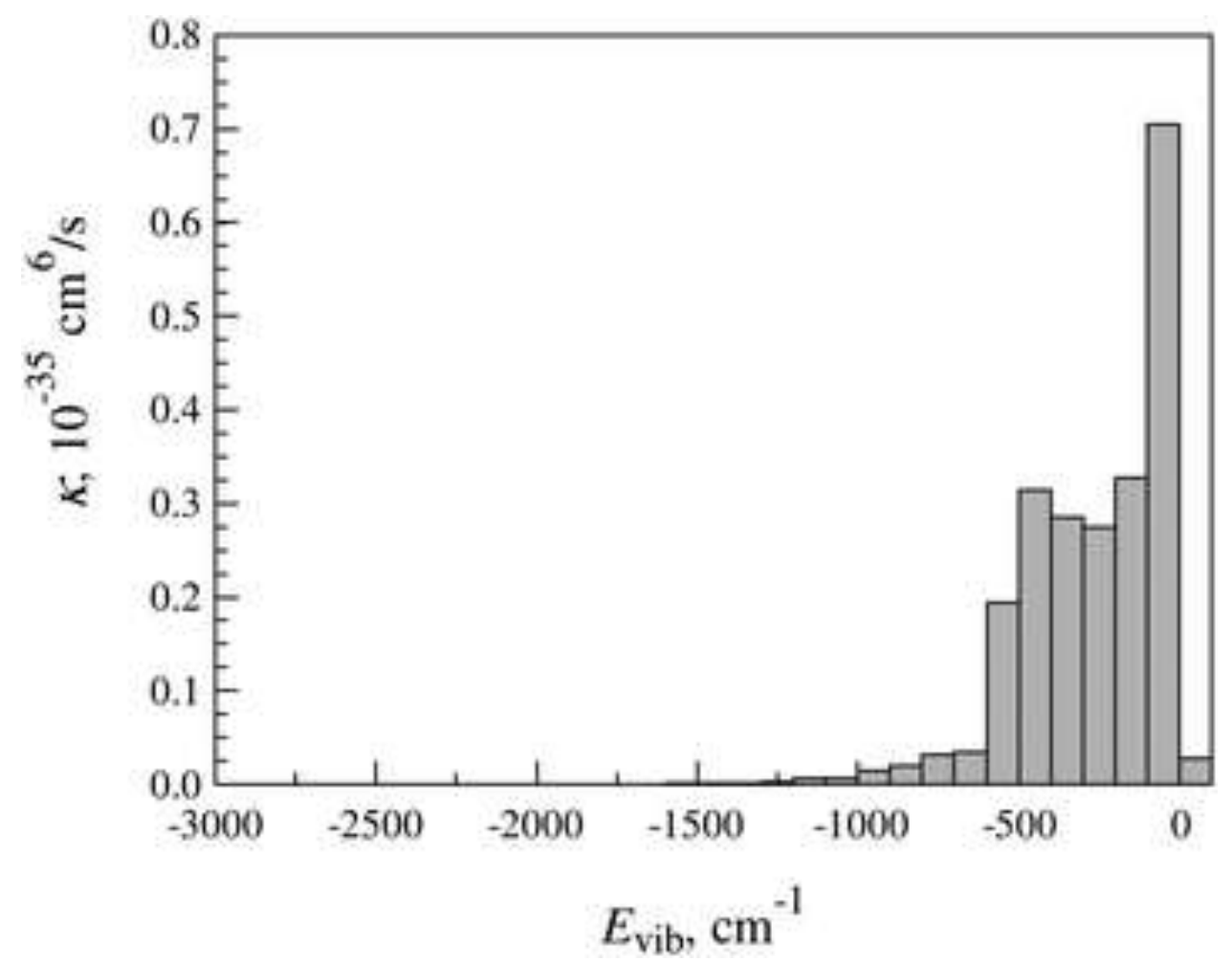

Fig. 5 Contributions of scattering resonances to the recombination rate coefficient $k$ as a function of vibrational energy. Only resonances localized in the well are included. Both symmetries are combined.

Further insight comes from analyzing where these resonances are (in terms of their energy) relative to the top of the centrifugal barrier. The effective barrier $\mathrm{E}^{\dagger}$ along the dissociative coordinate $\rho$ can be defined for given $\mathrm{J}$ and $\mathrm{K}_{\mathrm{a}}$ as the maximum value of the ground vibrational state in the two-dimensional eigenvalue problem solved for non-dissociative coordinates $\theta$ and $\varphi$. Since the PES of ozone has no activation barrier for $\mathrm{O}_{2}+\mathrm{O} \rightarrow \mathrm{O}_{3}$, the value of $\mathrm{E}^{\dagger}$ remains negative at lower levels of rotational excitation, forming a submerged "reef" rather than a barrier. At higher levels of rotational excitation $\mathrm{E}^{\dagger}$ shows up above the dissociation threshold. (The borderline cases $\mathrm{E}^{\dagger}=0$ are found at $\mathrm{J}=25, \mathrm{~K}_{\mathrm{a}}=0$, or $\mathrm{J}=20, \mathrm{~K}_{\mathrm{a}}=6$, or $\mathrm{J}=12, \mathrm{~K}_{\mathrm{a}}=8$, etc. The exact position of this border is sensitive to the shape of the PES. ${ }^{26}$ ) Thus, Fig. 6 gives correlation between the resonance lifetime $\Gamma_{i}$ and the offset of resonance energy from this effective barrier top: $\delta E_{i}=E_{i}$ $-\mathrm{E}^{\dagger}$. Color indicates contribution to the recombination rate coefficient $\kappa$. We see that some contribution, around $9 \%$, comes from resonances at energies within $50 \mathrm{~cm}^{-1}$ below the barrier top. These can be 
populated by tunneling only and, consequently, exhibit narrower widths, on the order of $\Gamma_{\mathrm{i}} \approx 10^{-2} \mathrm{~cm}^{-1}$. Resonances at energies within $150 \mathrm{~cm}^{-1}$ above the barrier top make the largest contribution to recombination. They are broader, $10^{-2}<\Gamma_{\mathrm{i}}<10 \mathrm{~cm}^{-1}$, and can be populated by redistribution of vibrational energy within the three modes of $\mathrm{O}_{3} *$, rather than tunneling.

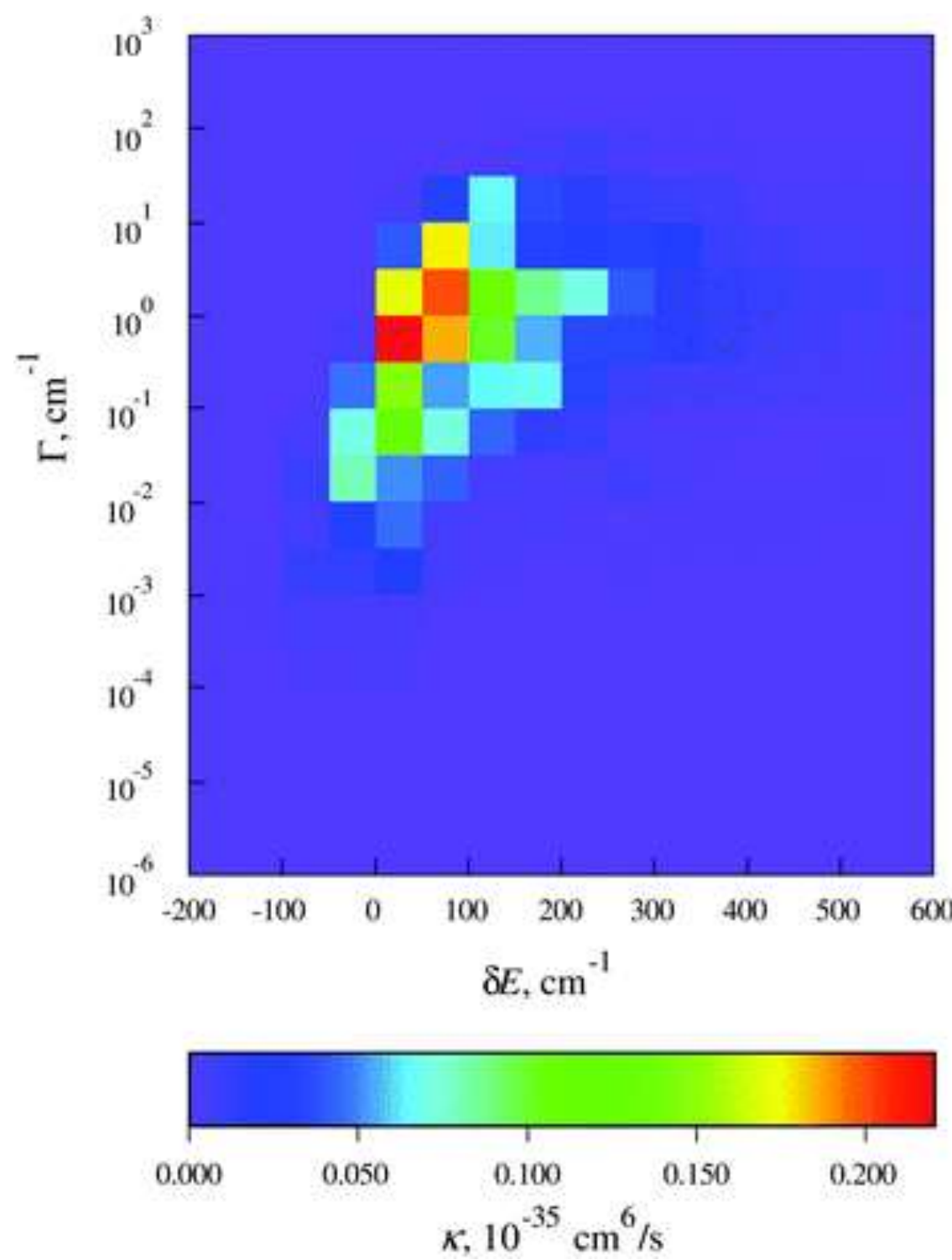

Fig. 6 Contributions of scattering resonances to the recombination rate coefficient $k$ as a function of resonance energy relative to the top of the centrifugal barrier $\left(\delta E_{i}\right.$ in the text) and the resonance width $\Gamma_{i}$.

Physical Chemistry Chemical Physics, No. 18 (2016): pg. 19194-19206. DOI. This article is @ Royal Society of Chemistry and permission has been granted for this version to appear in e-Publications@Marquette. Royal Society of Chemistry does not grant permission for this article to be further copied/distributed or hosted elsewhere without the express permission from Royal Society of Chemistry. 
Finally, Fig. 7 presents correlation between $\delta \mathrm{E}_{\mathrm{i}}$ and the probability of finding the system behind the centrifugal barrier, over the well region. This moiety, called here the well probability and denoted by $p_{w}$, is obtained by integrating the square modulus of wave function through the range $0 \leq \rho \leq \rho^{\dagger}$. In Fig. 7 we see that for typical resonances at energies slightly below the top of the centrifugal barrier or somewhat above it, this probability exceeds $\mathrm{p}_{\mathrm{w}}=0.7$. However, many higher energy resonances are more delocalized. Their contribution to the recombination process is not negligible, around $30 \%$. A schematic in Fig. 8 is used to demonstrate this concept. It shows examples of wave functions for three resonances: one sitting deep and behind the centrifugal barrier, one near the top of the barrier, and one significantly above the top of the barrier.

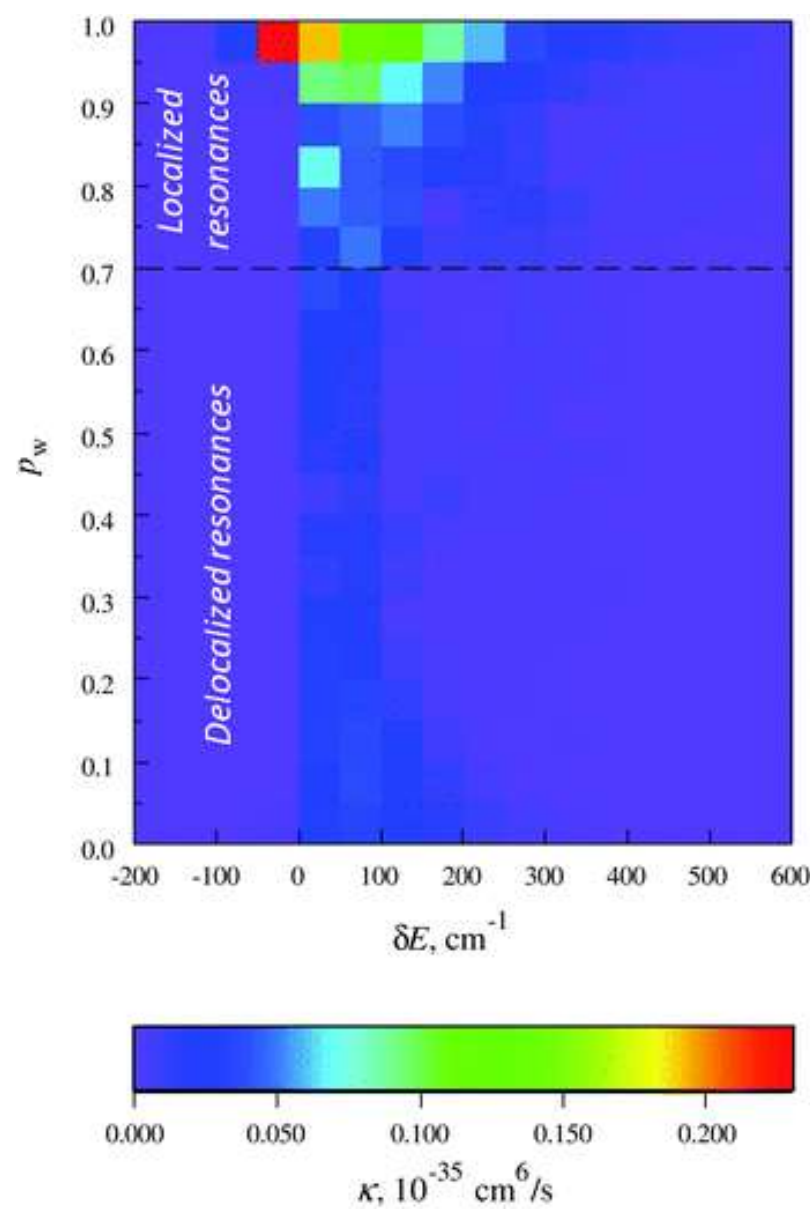

Fig. 7 Contributions of scattering resonances to the recombination rate coefficient $k$ as a function of resonance energy relative to the top of the centrifugal barrier $\left(\delta \mathrm{E}_{\mathrm{i}}\right)$

Physical Chemistry Chemical Physics, No. 18 (2016): pg. 19194-19206. DOI. This article is @ Royal Society of Chemistry and permission has been granted for this version to appear in e-Publications@Marquette. Royal Society of Chemistry does not grant permission for this article to be further copied/distributed or hosted elsewhere without the express permission from Royal Society of Chemistry. 
and the probability of the corresponding wave function over the well region. The boundary between resonances localized in the well and delocalized resonances is depicted with a dashed line at $\mathrm{p}_{\mathrm{w}}=0.7$.

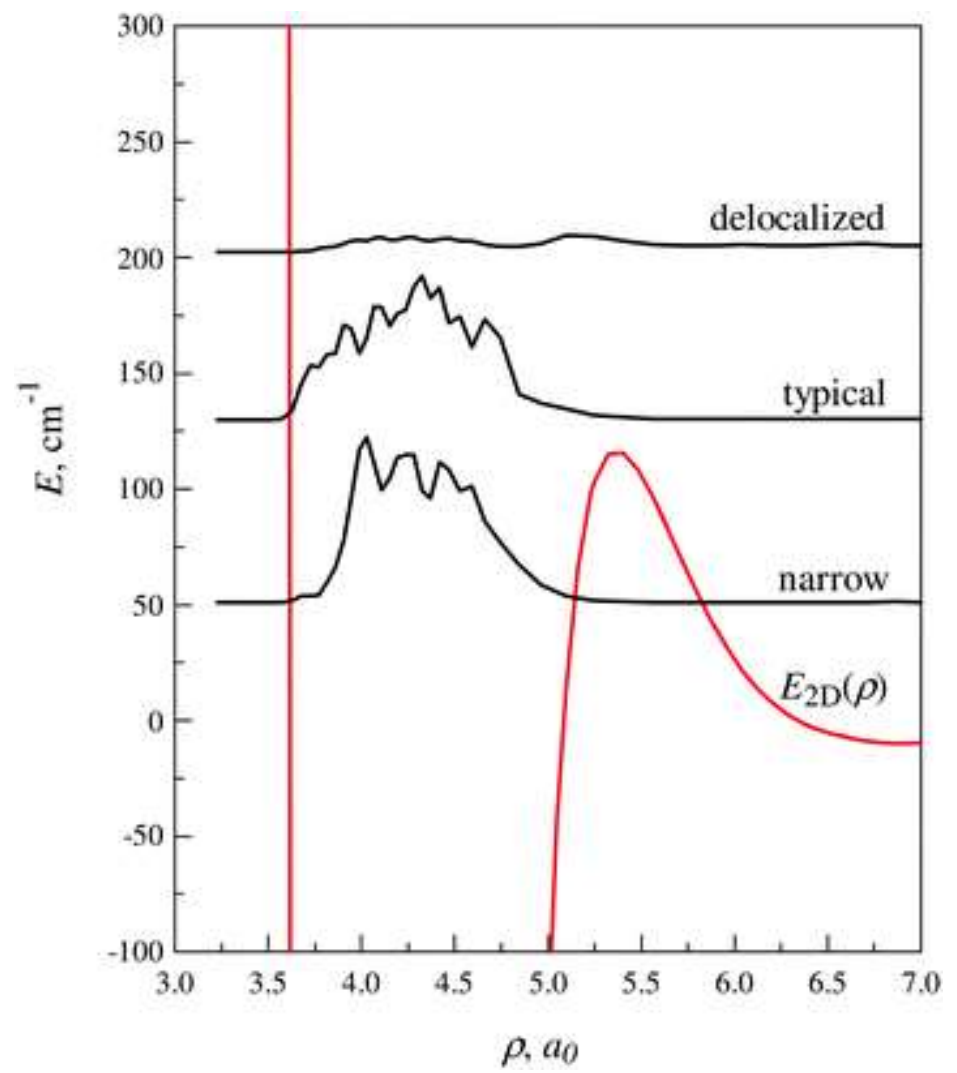

Fig. 8 Three types of resonances observed in our calculations: narrow resonance trapped behind the centrifugal barrier $\left(E=51.1 \mathrm{~cm}^{-1}, \Gamma=3.6 \times 10^{-4} \mathrm{~cm}^{-1}\right)$, typical resonance slightly near the barrier top $\left(E=130.0 \mathrm{~cm}^{-1}, \Gamma=0.16 \mathrm{~cm}^{-1}\right)$, and a highly delocalized state above the barrier $\left(E=202 \mathrm{~cm}^{-1}, \Gamma=15.5 \mathrm{~cm}^{-1}\right)$. The barrier top is at $115.7 \mathrm{~cm}^{-1}$, rotational excitation is $\mathrm{J}=32, \mathrm{~K}=0$.

The data presented in Fig. 1-7 can be summarized and interpreted in the following way: scattering resonances that participate in the recombination process represent upper bound states of nonrotating ozone $\left(600 \mathrm{~cm}^{-1}\right.$ below dissociation threshold) that are "lifted" by rotational excitation to energies above the dissociation threshold (around $100 \mathrm{~cm}^{-1}$ ), where these states can be populated from $\mathrm{O}+\mathrm{O}_{2}$. Most important contributions to the recombination process come from resonances at energies just slightly below or somewhat above the top of the centrifugal barrier $\left(-50<\delta \mathrm{E}_{\mathrm{i}}<150 \mathrm{~cm}^{-1}\right)$ and at moderate levels of rotational excitation $(8<\mathrm{J}<38)$. Widths of such resonances are not too small $\left(10^{-2}<\Gamma_{\mathrm{i}}<10 \mathrm{~cm}^{-1}\right)$, and their wave functions are 
localized dominantly over the covalent well, behind the centrifugal barrier $\left(p_{w}>0.7\right)$.

Indeed, on the lower energy side, resonances that sit too deep and behind the centrifugal barrier exhibit too narrow widths $\Gamma<10^{-2}$ $\mathrm{cm}^{-1}$, and contribute very little to recombination, according to eqn (9). On the higher energy side, the resonances at energies too far above the centrifugal barrier, although may be rather broad (even exceeding $\Gamma \approx 10 \mathrm{~cm}^{-1}$ ), they exhibit smaller stabilization cross sections because they are delocalized over the large range of $\rho$, which reduces the probability of their stabilization into the covalent well $\left(p_{w}<0.7\right)$. Moreover, at lower levels of rotational excitation $(\mathrm{J}<25)$ the top of the effective centrifugal barrier is still submerged below the dissociation limit $\left(E^{+}<0\right)$ which, again, makes resonances too delocalized. Finally, at higher levels of rotational excitation J $>40$ the Boltzmann factor shuts off the recombination process. So, it appears that only at moderate levels of rotational excitation and only at energies near the top of the centrifugal barrier the resonances of $\mathrm{O}_{3}$ are efficiently populated by $\mathrm{O}+\mathrm{O}_{2}$ collisions and are efficiently stabilized by Ar collisions. Widths of such resonances are neither too narrow nor too broad, as one can see from Fig. $1 \mathrm{~b}, 2$ and 6.

\section{III-B. Absolute value of the recombination rate coefficient}

The main (future, not immediate) goal of our efforts is to understand the anomalous isotope effect of ozone formation observed by the Mauersberger group and reported in a series of papers (see, for example ref. 7 and 8 , review articles ref. 1 and 2 and references therein). The majority of those studies were carried out at room temperature $\mathrm{T}=296 \mathrm{~K}$ and pressure $\mathrm{P}=200 \mathrm{Torr}$, in the Ar bath gas, which translates into $[\mathrm{M}]=6.53 \times 10^{18} \mathrm{~cm}^{-3}$. Under these conditions the total rate coefficient for ozone recombination is $\mathrm{K}^{\mathrm{TOT}}=42 \times 10^{-35}$ $\mathrm{cm}^{6} \mathrm{~s}^{-1} .^{43}$ According to the analysis of Troe $^{3}$ the contribution of the energy-transfer mechanism should be around $\mathrm{K}^{\mathrm{ET}}=23 \times 10^{-35} \mathrm{~cm}^{6} \mathrm{~s}^{-1}$ (close to $55 \%$ of the total rate). This is the value we are trying to reproduce by calculations.

Physical Chemistry Chemical Physics, No. 18 (2016): pg. 19194-19206. DOI. This article is @ Royal Society of Chemistry and permission has been granted for this version to appear in e-Publications@Marquette. Royal Society of Chemistry does not grant permission for this article to be further copied/distributed or hosted elsewhere without the express permission from Royal Society of Chemistry. 
The only other work, where the absolute value of the recombination rate coefficient obtained from quantum mechanics was reported, is the paper of Charlo and Clary. ${ }^{13}$ Their calculations gave $\mathrm{K}$ $=13 \times 10^{-35} \mathrm{~cm}^{6} \mathrm{~s}^{-1}$, although it is not entirely clear whether this value was taken directly from the dimensionally-reduced model, where the bending states are missing, or, it already includes a correction to account for the missing states (which would be a reasonable thing to do). Also, within a model based on classical trajectory simulations Schinke and Fleurat-Lessard ${ }^{4}$ were able to reproduce the overall experimental value of $\mathrm{K}^{\mathrm{TOT}}$ but empirically, by adjustment of stabilization efficiency (coefficient $\Delta=350 \mathrm{~cm}^{-1}$ in their theory). To the best of our knowledge, these are the only two theoretical predictions of the recombination rate coefficient available in the literature. In all other papers on ozone the workers either were interested in the ratio of the recombination rates for different isotopomers, or looked at other processes, such as atom exchange or ro-vibrational energy transfer (so, did not report the absolute value of recombination rate coefficient).

In our case the less certain component of recombination theory is the stabilization step, or, more precisely, the values of $\sigma^{\text {stab }}{ }_{\text {i. }}$. Several models developed in our previous work (as explained in Section II-C) are tested here by comparison vs. the experimental value of $\mathrm{K}^{\mathrm{ET}}$. The results of these tests are presented in Table 1 . We see that all stabilization models give the recombination rate coefficient $\mathrm{k}$ of correct order of magnitude. Namely, the first column in Table 1 shows that the values of $\mathrm{k}$ obtained using SM1a and SM1b models fall between the minimum and maximum limits predicted by SM2. This makes sense, since SM1a and SM1b were constructed to represent stabilization cross sections on average, while SM2 represents the possible range. As for comparison with experiment we see that our predicted rate coefficients are somewhat smaller. Even the upper limit given by the SM2 model, $\mathrm{k}$ $\approx 16.1 \times 10^{-35} \mathrm{~cm}^{6} \mathrm{~s}^{-1}$, is only $70 \%$ of the experimental value of $\mathrm{K}^{\mathrm{ET}}$ (although it is very close to the result of $\mathrm{Clary}^{13}$ ).

Table 1. Recombination rate constant $\mathrm{k}\left(10^{-35} \mathrm{~cm}^{6} \mathrm{~s}^{-1}\right)$ for the energy transfer mechanism
Model
Localized resonances $\underline{\underline{a}}$
All resonances $\underline{b}$

a Includes states with fraction in the well more than $70 \%$. b Includes all states where contribution of delocalized states is multiplied by fraction.

Physical Chemistry Chemical Physics, No. 18 (2016): pg. 19194-19206. DOI. This article is @ Royal Society of Chemistry and permission has been granted for this version to appear in e-Publications@Marquette. Royal Society of Chemistry does not grant permission for this article to be further copied/distributed or hosted elsewhere without the express permission from Royal Society of Chemistry. 
NOT THE PUBLISHED VERSION; this is the author's final, peer-reviewed manuscript. The published version may be accessed by following the link in the citation at the bottom of the page.

$\begin{array}{lll}\text { Model } & \text { Localized resonances } \underline{\underline{a}} & \text { All resonances } \underline{\underline{b}} \\ \text { SM1a } & 13.4 & 19.1 \\ \text { SM1b } & 9.4 & 12.2 \\ \text { SM2 } & 5.6-16.1 & 8.3-22.7 \\ \text { SM2' } & 8.1-19.4 & 11.6-27.0 \\ \text { Experiment } & & 23\end{array}$

Trying to find the missing piece we noticed that the lowest energy scattering resonances $\mathrm{O}^{(\mathrm{i})}{ }_{3}$ do not contribute to recombination. Qualitatively, they sit so deep and behind the centrifugal threshold that they can't be populated in a typical $\mathrm{O}_{2}+\mathrm{O}$ collision, just because heavy particles can't tunnel through wide barriers (see Fig. 8). Quantitatively, in the limit $\Gamma_{i} \ll\left(k^{\text {stab }_{i}}+k^{\text {diss }_{i}}\right)[M]$ the contribution of each resonance, according to eqn (9), simplifies to the following expression: $\mathrm{K}_{\mathrm{i}} \approx 1 / 2 \Gamma_{\mathrm{i}} \mathrm{K}_{\mathrm{eq}}\left(\mathrm{E}_{\mathrm{i}}\right) /[\mathrm{M}]$. This shows clearly that resonances with negligible widths $\Gamma_{i}$ make negligible contributions to recombination. Thus, they should be considered as bound states, rather than scattering resonances. Removing these states from the list of resonances does not reduce the value of the recombination rate coefficient much, but this modification "lifts" the bound state threshold, leading to more efficient stabilization of the remaining (higher energy) scattering resonances, since now the required energytransfer $\Delta \mathrm{E}$ is effectively reduced for them. We found that (at this pressure) the resonances with widths below $\Gamma_{\mathrm{i}} \approx 10^{-2} \mathrm{~cm}^{-1}$ can be considered as effectively bound states. This adjustment, which represents an improved version of SM2, called SM2' hereafter, permits increasing the maximum limit of the recombination rate coefficient to $\mathrm{K}$ $\approx 19.4 \times 10^{-35} \mathrm{~cm}^{6} \mathrm{~s}^{-1}$ (bottom of the first column in Table 1 ), which is about $84 \%$ of the experimental value of $\mathrm{K}^{\mathrm{ET}}=23 \times 10^{-35} \mathrm{~cm}^{6} \mathrm{~s}^{-1}$, and is still not quite sufficient.

Searching for the still missing piece we realized that we took into consideration, so far, only resonances that are localized mostly over the covalent well, inside the centrifugal barrier, rather than outside (see Fig. 8). As we showed in Section III-A above, for typical resonances at energies slightly below the top of the centrifugal barrier or slightly above it, the value of well probability $\mathrm{p}_{\mathrm{w}}$ exceeds 0.7 or so. Consequently, the results given in the first column of Table 1 were obtained including only localized resonances, with $p_{w}>0.7$. However, many higher energy resonances are more delocalized, characterized by permission has been granted for this version to appear in e-Publications@Marquette. Royal Society of Chemistry does not grant permission for this article to be further copied/distributed or hosted elsewhere without the express permission from Royal Society of Chemistry. 
$\mathrm{p}_{\mathrm{w}}<0.7$. Although energies and lifetimes of these states are available from our calculations, it appears that including them into consideration of recombination kinetics is not straightforward, because our stabilization models (SM1 and SM2) were set up for the localized states only. ${ }^{20,22}$ From ref. 19 we know that transitions from the outside of the centrifugal barrier into the states localized in the well are very weak. So, using SM1 and SM2 for delocalized states would be incorrect and would, certainly, overestimate the value of the recombination coefficient $\mathrm{k}$ (we checked this by calculations).

It makes sense, however, to use SM1 and SM2 for only a portion of the delocalized resonance, namely, for that piece of it that sits inside of the centrifugal barrier. This is equivalent to multiplying stabilization cross section by the well probability $\mathrm{p}$. So, for the localized states (characterized by $p_{w}>0.7$ ) we use SM1 and SM2 straight, whereas for delocalized states $\left(p_{w}<0.7\right)$ we reduce the value of stabilization cross section proportionally to the well probability $p$. Recombination rate coefficients computed in this way, with delocalized states included, are given in the second column of Table 1 . They are somewhat higher than those in the first column of Table 1. Predictions of SM1a and SM1b are still somewhat below the experimental value of $\mathrm{K}^{\mathrm{ET}}$, but the upper limit of the SM2 model with delocalized states included is now $\mathrm{K}=22.7 \times 10^{-35} \mathrm{~cm}^{6} \mathrm{~s}^{-1}$, which almost reaches the experimental value of $\mathrm{K}^{\mathrm{ET}}=23 \times 10^{-35} \mathrm{~cm}^{6} \mathrm{~s}^{-1}$. Finally, the upper limit of $\mathrm{SM}^{\prime}$ is now $\mathrm{K}=27.0 \times 10^{-35} \mathrm{~cm}^{6} \mathrm{~s}^{-1}$, which is above the experimental value of $\mathrm{K}^{\mathrm{ET}}$. So, in this final form of our theory, the experimental value of $\mathrm{K}^{\mathrm{ET}}$ is within the ranges predicted by the SM2' model.

The following conclusion can be drawn from the data presented in Table 1. In order to reproduce the experimental rate coefficient for ozone recombination we had to take delocalized resonances into consideration. It is also important to treat the low-energy resonances as bound states, but this is not sufficient by itself. Only when those two effects are both included, the experimental value of the recombination rate coefficient is recovered by the SM2' model of stabilization. In what follows we will use this approach as the working model, but, for comparison, we will also present results obtained using the simplest SM1a model (with delocalized states included), which gives the rate coefficient just $17 \%$ below the experimental value of $\mathrm{K}^{\mathrm{ET}}$. permission has been granted for this version to appear in e-Publications@Marquette. Royal Society of Chemistry does not grant permission for this article to be further copied/distributed or hosted elsewhere without the express permission from Royal Society of Chemistry. 
NOT THE PUBLISHED VERSION; this is the author's final, peer-reviewed manuscript. The published version may be accessed by following the link in the citation at the bottom of the page.

Note that in Fig. 1-3, 6 and 7 were generated using SM1a with delocalized states included. Fig. 4 and 5 were generated using SM1a without delocalized states (since it is harder to make connection between the highly excited delocalized scattering resonances and the bound states of a non-rotating ozone molecule).

\section{III-C. Pressure dependence}

Pressure dependence of the third-order rate coefficient for ozone formation is known to be weak. So, it is usual to plot, as a function of pressure, the product $\mathrm{K} \times[\mathrm{M}]$, which represents the second-order rate coefficient for recombination of $\mathrm{O}_{2}$ with $\mathrm{O}$. Its pressure dependence is expected to be roughly linear. Fig. 9 presents experimental data from ref. 36 and 37 in a broad range of pressure values, for three representative temperatures. We focus on frame $b$ of this figure, since that temperature, $\mathrm{T}=300 \mathrm{~K}$, is very close to the temperature in experiments of the Mauersberger group. For completeness, the value of the recombination rate coefficient given by Mauersberger and co-workers ${ }^{43}$ is also shown in Fig. 9b, at low pressure $\mathrm{P}=200$ Torr. 
NOT THE PUBLISHED VERSION; this is the author's final, peer-reviewed manuscript. The published version may be accessed by following the link in the citation at the bottom of the page.
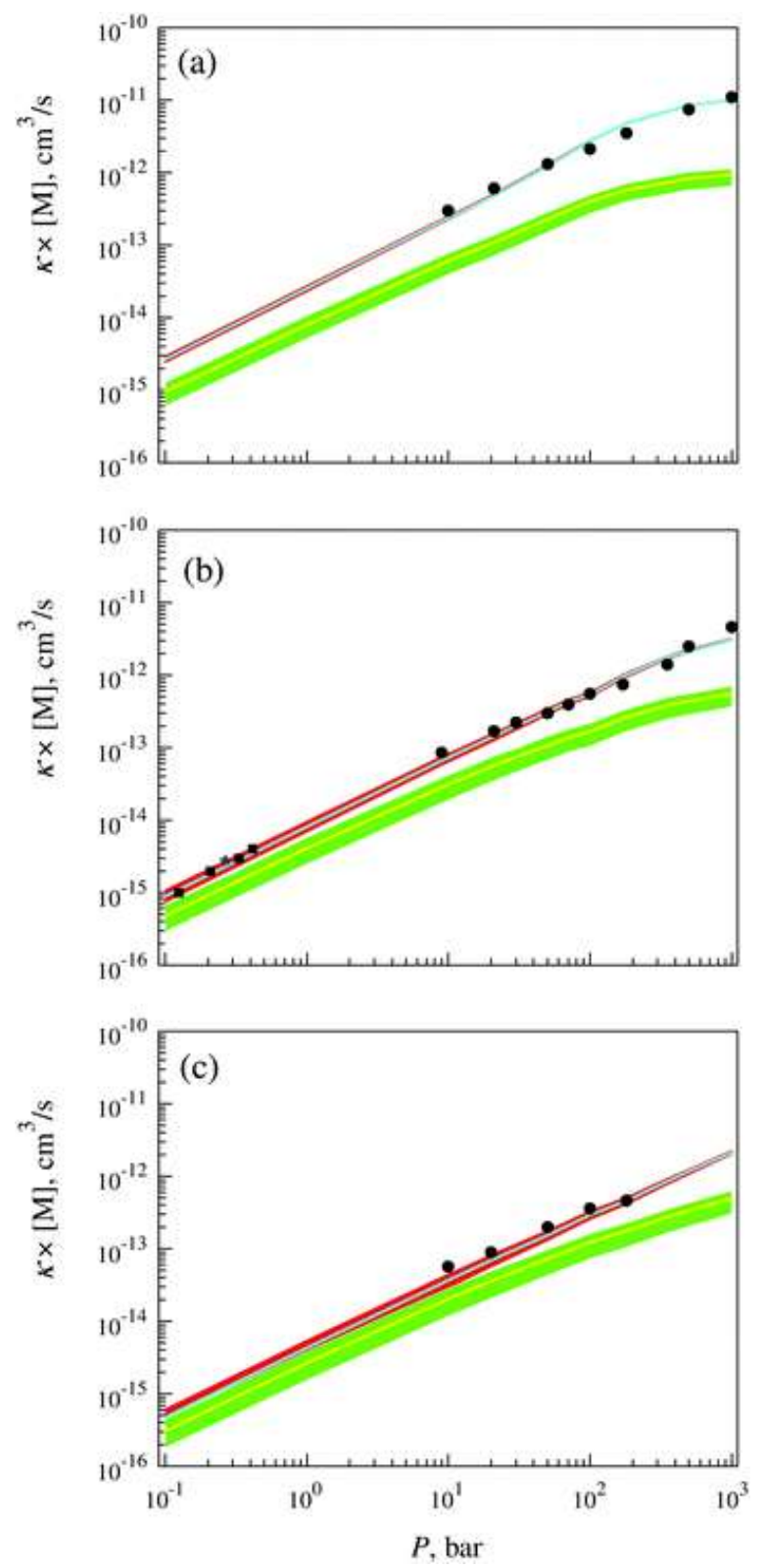

Fig. 9 Pressure dependence of the second-order recombination rate coefficient $k \times$ [M] for three temperatures: (a) $213 \mathrm{~K}$, (b) $300 \mathrm{~K}$ and (c) $373 \mathrm{~K}$. Yellow line with green range corresponds to the energy transfer mechanism alone, while blue line with red range represents the total rate coefficient, with chaperon contribution added. Black symbols depict experimental data: circles (ref. 44), star (ref. 43) and squares (ref. 45 ).

\section{Our predictions of $\mathrm{K}^{\mathrm{ET}} \times[\mathrm{M}]$, computed using the SM1a and} SM2' models of stabilization, are shown on these figures too (yellow line and green range). As expected, the results of the SM1a model are within the range of the SM2' model, and show the same pressure

Physical Chemistry Chemical Physics, No. 18 (2016): pg. 19194-19206. DOI. This article is @ Royal Society of Chemistry and permission has been granted for this version to appear in e-Publications@Marquette. Royal Society of Chemistry does not grant permission for this article to be further copied/distributed or hosted elsewhere without the express permission from Royal Society of Chemistry. 
dependence. The dependence starts roughly linear, but it falls off at higher pressure. This behavior is typical to the energy-transfer (Lindeman) mechanism of recombination. It is explained by competition between two terms in the denominator of eqn (9), namely, between the spontaneous decay of scattering resonances and their collision-induced stabilization. At high pressure one can neglect $\Gamma_{i}$ in the denominator of eqn (9), which leads to $\kappa_{i} \times[M] \approx 1 / 2 \Gamma_{i} K_{\text {eq }}\left(E_{i}\right)$ for each resonance. This means that the value of $K^{\mathrm{ET}} \times[\mathrm{M}]$ stops increasing with pressure, it decreases, just as shown in Fig. 9. Such behavior was also observed by Marcus and co-workers in their model of ozone formation, ${ }^{46}$ and by Pack et al. ${ }^{47}$ in their studies of the $\mathrm{Ne}+$ $\mathrm{Ne}+\mathrm{H} \rightarrow \mathrm{Ne}_{2}+\mathrm{H}$ recombination process.

Note, however, that the experimental data in Fig. 9 hardly show any decrease. As discussed above, the analysis of Troe ${ }^{3}$ indicates that in the experiment the recombination process involves both the energytransfer and the radical-complex (chaperon) mechanisms. Therefore, in order to compare with experiment, we added to our $\mathrm{k} \times[\mathrm{M}]$, predicted here by calculations, the contribution of radical-complex $\mathrm{K}^{\mathrm{RC}}$ $\times[\mathrm{M}]$, determined by $\mathrm{Troe}^{3}$ from the analysis of experimental data. The total rate is shown by blue line with red range in Fig. 9 , and we see that it is in good agreement with experiment, both in terms of the absolute value and pressure dependence, in a broad range.

Moreover, our calculations show similarly good agreement with experimental data for pressure dependence at slightly elevated and slightly reduced temperatures: $\mathrm{T}=373 \mathrm{~K}$ and $\mathrm{T}=213 \mathrm{~K}$, respectively. This is illustrated by Fig. 9a and c. The fall off is more pronounced at lower temperature, and is barely visible at higher temperature. In either case, the total of energy-transfer and radical-complex contributions agree well with the experiment in a broad range of pressure values, and for all three values of temperature.

\section{III-D. Temperature dependence}

Initially, we did not plan to study temperature dependence of the recombination rate coefficient. For this, strictly speaking, one has to compute temperature dependence of stabilization cross sections, but we did not really do that. Our previous mixed quantum/classical calculations of energy-transfer ${ }^{20-22}$ were carried out at room

Physical Chemistry Chemical Physics, No. 18 (2016): pg. 19194-19206. DOI. This article is @ Royal Society of Chemistry and permission has been granted for this version to appear in e-Publications@Marquette. Royal Society of Chemistry does not grant permission for this article to be further copied/distributed or hosted elsewhere without the express permission from Royal Society of Chemistry. 
temperature only. However, reasonable agreement with experiment in all three frames of Fig. 9, that cover the temperature range $213 \leq \mathrm{T} \leq$ $373 \mathrm{~K}$, indicates that we can try to explore the temperature dependence, at least in a narrow range of couple hundred degrees Kelvin. Furthermore, quantum calculations of temperature dependence of stabilization rate coefficients by Charlo and Clary ${ }^{12}$ showed that those depend only weakly on temperature, even in a much broader temperature range. Similar conclusions were drawn by Ivanov and Schinke, ${ }^{48,49}$ based on classical trajectory simulations.

So, we decided to check what temperature dependence is predicted by our calculations. Fig. 10 shows experimental data available for the third-order recombination rate coefficient through a broad range of temperatures, as summarized by Troe. ${ }^{3}$ Our predictions, obtained using SM1a and SM2' models for stabilization, are also shown in Fig. 10. Yellow line with green range is used for $k(T)$ based on the energy-transfer mechanism alone, while blue line with red range is used for the total of our $\mathrm{k}(\mathrm{T})$ plus the $\mathrm{K}^{\mathrm{RC}}(\mathrm{T})$ contribution, as reported by Troe. ${ }^{3}$ We see that temperature dependence of the total recombination rate is in very good agreement with experiments. The temperature dependence of the $\mathrm{K}^{\mathrm{ET}}(\mathrm{T})$ alone is not available from the experiment, but it can be estimated (extrapolated) from the hightemperature data, where the contribution of the radical-complex mechanism is expected to vanish. This extrapolation is shown by black line in Fig. 10. We see that our prediction of temperature dependence for the energy-transfer mechanism alone is also in reasonable agreement with experimental information. Better agreement is hard to achieve, due to the nature of extrapolation, but also due to a significant spread of experimental data in the high temperature range, as one can see in Fig. 10. 
NOT THE PUBLISHED VERSION; this is the author's final, peer-reviewed manuscript. The published version may be accessed by following the link in the citation at the bottom of the page.

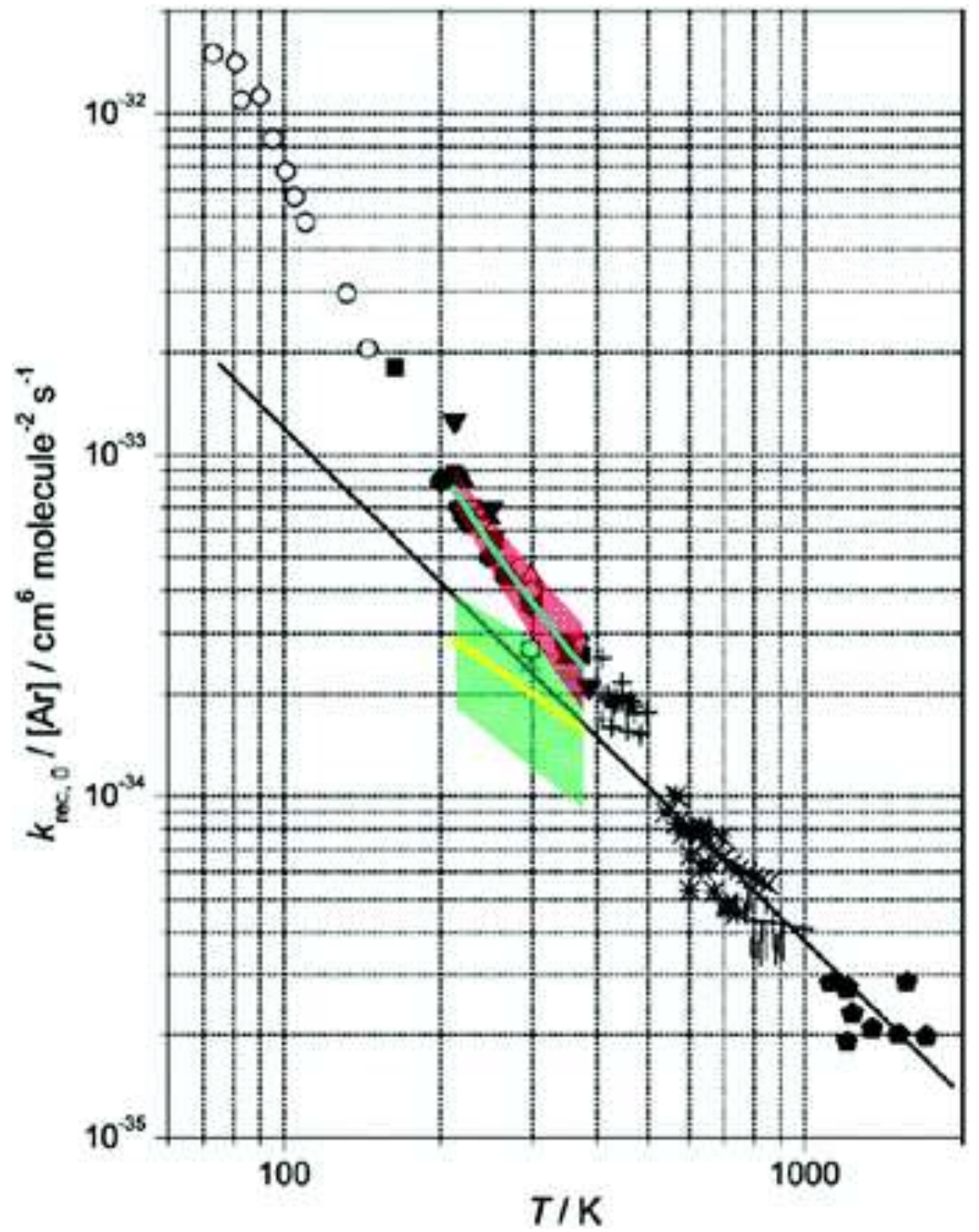

Fig. 10 Temperature dependence of the recombination rate coefficient $K$ at $P=0.1$ bar. Yellow line with green range corresponds to the energy transfer mechanism alone, while blue line with red range represents the total rate coefficient, with chaperon contribution added. The original figure, containing combined experimental data, was taken from the paper by Troe, ref. 3 . The long black line is an experiment-based estimation of the ET rate coefficient.

It is usual to fit experimental or theoretical data for temperature dependencies by $\mathrm{T}^{-\mathrm{n}}$ functions. We also did this, using different models for the stabilization process. Our results are summarized in Table 2, together with other theoretical predictions from the literature, and available experimental data. The first column of Table 2 gives the values of $\mathrm{n}$ for the energy-transfer mechanism alone. Predictions of SM1a and SM2 models are slightly below the experimental value of $n$

Physical Chemistry Chemical Physics, No. 18 (2016): pg. 19194-19206. DOI. This article is @ Royal Society of Chemistry and permission has been granted for this version to appear in e-Publications@Marquette. Royal Society of Chemistry does not grant permission for this article to be further copied/distributed or hosted elsewhere without the express permission from Royal Society of Chemistry. 
$=1.5$, while prediction of SM1b is slightly above it. A larger deviation from the experimental value of $n$ was observed in the work of Grebenshchikov and Shinke ${ }^{16}$ where $n=2.1$ was reported, which is close to the temperature dependence of the total recombination rate coefficient. Charlo and Clary $^{13}$ had negative temperature dependence, $\mathrm{n}=-0.64$, due to the artifact of the older potential energy surface. Schinke and Fleurat-Lessard ${ }^{4}$ obtained $n=1.5$, but only after empirical adjustment of the damping coefficient in their classical (trajectory based) approach.

Table 2. Temperature dependence $T^{-n}$ of the recombination rate constant

ET mechanism

Localized

Model resonances $\underline{a}$
All resonances $\underline{b}$
ET + RC mechanisms

Localized All

resonances $\underline{\underline{a}}$ resonances $\underline{\underline{b}}$

a Includes states with fraction in the well more than $70 \%$. b Includes all states where contribution of delocalized states is multiplied by fraction.

$\begin{array}{lllll}\text { SM1a } & 0.96 & 1.09 & 2.16 & 2.10 \\ \text { SM1b } & 1.55 & 1.72 & 2.66 & 2.65 \\ \text { SM2 } & 1.00-1.37 & 1.12-1.49 & 2.16-2.69 & 2.09-2.62 \\ \text { SM2' } & 0.86-1.15 & 0.98-1.28 & 2.01-2.48 & 1.95-2.42 \\ \text { Ref. 16 } & & 2.1 & & \\ \text { Ref. 4 } & & 1.5 & & \\ \text { Ref. 13 } & & -0.64 & & 2.22(2.7)\end{array}$

The second column in Table 2 reports the values of $n$ obtained for temperature dependence of the total recombination rate coefficient which, again, includes our computed contribution of the energytransfer mechanism, plus the contribution $\mathrm{K}^{\mathrm{RC}}(\mathrm{T})$ of the radicalcomplex mechanism as reported by Troe. ${ }^{3}$ The experimental value of $n$ $=2.2$ is in the ranges predicted by SM2 and SM2'. The prediction of SM1a is somewhat lower than the experimental value, while prediction of SM1b is somewhat above it. The experimental value of $n=2.7$ reported in the earlier work ${ }^{50}$ is closer to the prediction of SM1b and the upper limit of SM2.

\section{III-E. Discussion}

Comparison of our results vs. experimental data is very good. We should admit, however, that the less certain component of our theory, the stabilization step, is still described approximately, by a 
simple analytic energy transfer model (several variations of which have been tested above). Alternatively, one may wish to use accurately computed stabilization and dissociation cross sections for each individual resonance, which could be regarded as the exact approach to the problem. In fact, we did such calculations in our earlier work, but only for the dimensionally-reduced model of an ozone molecule (with bending states omitted) and within the framework of the mixed quantum/classical theory. Similar quantum/classical calculations for stabilization of individual ro-vibrational states within the full-dimensional description of the ozone molecule would be extremely demanding, while the full-quantum scattering calculations would be computationally unaffordable. So, for now, we decided to explore what can be learned using those simpler models for the stabilization step.

Although in this work we tested several models of the energy transfer, note that we did not tune any parameters in these models (e.g., trying to reproduce experimental data). Instead, we explored all possible sources of contributions to the recombination process. But, in principle, someone may want to ask a question: could it be that slight variation of model parameters would permit reproducing the total experimental rate coefficient by the energy-transfer mechanism alone, without any involvement of the chaperon mechanism? Well, in order to reach the value of $\mathrm{K}^{\text {TOT }}=42 \times 10^{-35} \mathrm{~cm}^{6} \mathrm{~s}^{-1}$ within the current approach and using SM1a (with delocalized states included) we would have to increase the value of $\sigma^{\text {stab }}{ }_{0}$ in eqn (11) by a factor of 2.5 , and, it would be problematic to find a justification for this. In fact, our stabilization cross sections are already rather large. Comparisons can be made with the thermal stabilization rate coefficient reported by Clary, ${ }^{12} 4.92 \times 10^{-11} \mathrm{~cm}^{3} \mathrm{~s}^{-1}$, and with the energy transfer function reported by Schinke (see Fig. 2 in ref. 49). We checked and found that the corresponding numbers in our calculations are larger than those of Clary by a factor of three, and, larger by a factor of four than those of Schinke. So, we would be very hesitant to "tune" stabilization cross section without any justification, just in order to fit the experimental value of $\mathrm{K}^{\text {TOT }}$. Inclusion of delocalized resonances, and treating narrow resonances as bound states, permitted us to reach the level of experimental $\mathrm{K}^{\mathrm{ET}}$, but not the level of experimental $\mathrm{K}^{\mathrm{TOT}}$. We conclude that within the energy-transfer mechanism, we can't find any other possible source of additional contribution to recombination.

Physical Chemistry Chemical Physics, No. 18 (2016): pg. 19194-19206. DOI. This article is @ Royal Society of Chemistry and permission has been granted for this version to appear in e-Publications@Marquette. Royal Society of Chemistry does not grant permission for this article to be further copied/distributed or hosted elsewhere without the express permission from Royal Society of Chemistry. 
Moreover, we checked and found that a straightforward increase of $\sigma^{\text {stab }}{ }_{0}$ by a factor of 2.5 does not resolve all problems, because it leads to incorrect pressure and temperature dependencies. We tried this, and it appears that the resultant pressure dependence would exhibit a pronounced fall-off in the high-pressure regime, which contradicts with experiments. Quantitatively, at pressure $\mathrm{P}=10^{3} \mathrm{bar}$ the rate coefficient would be 3.1 times smaller than the experimental value. The temperature dependence would also be way too weak, with $\mathrm{n}=1.06$.

Furthermore, recall that as we explained above, in the highpressure limit eqn (9) simplifies, giving $K_{i} \times[M] \approx 1 / 2 \Gamma_{i} K_{e q}\left(E_{i}\right)$ which is independent of $\mathrm{k}^{\mathrm{stab}_{i}}$ at all! So, it does not matter which stabilization model is used, and how accurate it is. If the resonances (number of states, their energies and lifetimes) are computed using accurate treatment of $\mathrm{O}_{3}$, then this information alone sets up the high-pressure limit of the recombination rate, and there is no need to consider the $\mathrm{Ar}$ $+\mathrm{O}_{3}$ collision process. Since here the treatment of resonances in $\mathrm{O}_{3}$ is rather accurate, but the high-pressure rate coefficient is still 3.1 times smaller than the experiment, it means that there must be some additional contribution to recombination, other than the energy transfer mechanism. From our point of view this is the strongest argument in support of the chaperon mechanism.

So, it appears that in order to reproduce experimental data one must involve the chaperon mechanism, as we did here, using the fitting parameters of Troe.

\section{Conclusions}

In this paper we presented rigorous calculations and a detailed analysis of scattering resonances in ozone, for a broad range of rotational excitations. We adopted a recently developed accurate potential energy surface, ${ }^{26}$ and developed an efficient method for calculations of ro-vibrational energies, wave functions and resonance lifetimes ${ }^{23}$ (using hyper-spherical coordinates, the sequential diagonalization/truncation approach, grid optimization and complex absorbing potential). The distribution of resonance energies and lifetimes was discussed, as well as their rotational and vibrational content, and even other interesting features, such as positions of 
resonances with respect to the centrifugal barrier (both energetically and in terms of the probability distribution). Correlations between many of these properties were visualized with emphasis on the contribution of resonances into the recombination process.

This accurate information was augmented by the energytransfer models derived earlier for stabilization and dissociation of scattering resonances, ${ }^{20,22}$ in order to predict the absolute value of the recombination rate coefficient and determine its pressure and temperature dependencies. Our results offer strong support for the work of Troe, ${ }^{3}$ who argued that the energy transfer mechanism of recombination, the Lindeman mechanism, is just one of two mechanisms forming ozone. The recombination rate coefficient that we obtained for the energy-transfer mechanism at room temperature in $\mathrm{Ar}$ bath gas (using the simplest stabilization model SM1a) is about $45 \%$ of the total rate coefficient measured in the experiment. An alternative version of the stabilization model, $\mathrm{SM} 2^{\prime}$, gives the range of values 30$63 \%$. These match nicely with the conclusion of Troe, who derived that it should be close to 55\%. Furthermore, both experimentally determined pressure dependence of the rate coefficient (in a broad range) and its temperature dependence (in the narrower range) are reproduced well, if we add to our data the contribution of the chaperon mechanism, as derived by Troe.

In our calculations we found that it is important to include broader delocalized resonances at higher energies into consideration, since their contribution is not negligible. In the future, a model for the stabilization of such resonances, more accurate than the one adopted here, is desirable. It was also important to treat the low-energy narrow resonances (trapped deep and behind the centrifugal barrier) as bound states, which increases stabilization rates for the most important resonances at energies near the top of the barrier. In the future, one could try to implement solution of the master equation, in order to have these effects included automatically and more rigorously.

The accurate description of the energy-transfer mechanism of ozone formation is important for understanding anomalous enrichments of various isotopomers of ozone (there are 36 isotopically distinct variants of this reaction, see Table 1 in ref. 8 ). Our next step

Physical Chemistry Chemical Physics, No. 18 (2016): pg. 19194-19206. DOI. This article is @ Royal Society of Chemistry and permission has been granted for this version to appear in e-Publications@Marquette. Royal Society of Chemistry does not grant permission for this article to be further copied/distributed or hosted elsewhere without the express permission from Royal Society of Chemistry. 
will be to repeat, for several isotopic substitutions, all the calculations reported in this paper, hoping that the isotope effects will emerge in calculations, which would help understand its origin.

\section{Acknowledgements}

Richard Dawes is gratefully acknowledged for sharing his new PES of ozone. This research was supported by the NSF Atmospheric Chemistry Program, Division of Atmospheric Sciences, grant number AGS-1252486. This research used resources of the National Energy Research Scientific Computing Center, which is supported by the Office of Science of the U.S. Department of Energy under Contract No. DE-AC02-05CH11231. Alexander Teplukhin acknowledges support from Arthur J. Schmitt Leadership Fellowship.

\section{References}

${ }^{1}$ K. Mauersberger, D. Krankowsky, C. Janssen and R. Schinke, Adv. At., Mol., Opt. Phys., 2005, 50, 1-54.

${ }^{2}$ R. Schinke, S. Y. Grebenshchikov, M. V. Ivanov and P. Fleurat-Lessard, Annu. Rev. Phys. Chem., 2006, 57, 625-661.

${ }^{3}$ K. Luther, K. Oum and J. Troe, Phys. Chem. Chem. Phys., 2005, 7, 27642770.

${ }^{4}$ R. Schinke and P. Fleurat-Lessard, J. Chem. Phys., 2005, 122, 094317.

${ }^{5}$ M. V. Ivanov and R. Schinke, J. Chem. Phys., 2006, 124, 104303.

$6 \mathrm{M}$. H. Thiemens, Science, 1999, 283, 341-345.

${ }^{7} \mathrm{~K}$. Mauersberger, B. Erbacher, D. Krankowsky, J. Guenther and R. Nickel, Science, 1999, 283, 370-372.

${ }^{8} \mathrm{C}$. Janssen, J. Guenther, K. Mauersberger and D. Krankowsky, Phys. Chem. Chem. Phys., 2001, 3, 4718-4721.

${ }^{9}$ D. Babikov, B. K. Kendrick, R. B. Walker, R. T. Pack, P. Fleurat-Lesard and R. Schinke, J. Chem. Phys., 2003, 118, 6298-6308.

${ }^{10}$ D. Babikov, B. K. Kendrick, R. B. Walker, R. T. Pack, P. Fleurat-Lesard and R. Schinke, J. Chem. Phys., 2003, 119, 2577-2589.

${ }^{11}$ D. Babikov, B. K. Kendrick, R. B. Walker, R. Schinke and R. T. Pack, Chem. Phys. Lett., 2003, 372, 686-691.

${ }^{12}$ D. Charlo and D. C. Clary, J. Chem. Phys., 2002, 117, 1660-1672.

${ }^{13}$ D. Charlo and D. C. Clary, J. Chem. Phys., 2004, 120, 2700.

${ }^{14} \mathrm{~T}$. Xie and J. M. Bowman, Chem. Phys. Lett., 2005, 412, 131-134.

${ }_{15}$ M. V. Ivanov and R. Schinke, Mol. Phys., 2010, 108, 259-268.

${ }^{16}$ S. Y. Grebenshchikov and R. Schinke, J. Chem. Phys., 2009, 131, 181103.

${ }_{17}$ M. V. Ivanov and D. Babikov, J. Chem. Phys., 2011, 134, 144107.

${ }^{18}$ M. V. Ivanov and D. Babikov, Chem. Phys. Lett., 2012, 535, 173-176.

${ }^{19}$ M. V. Ivanov and D. Babikov, J. Chem. Phys., 2011, 134, 174308. 
NOT THE PUBLISHED VERSION; this is the author's final, peer-reviewed manuscript. The published version may be accessed by following the link in the citation at the bottom of the page.

20M. V Ivanov and D. Babikov, J. Chem. Phys., 2012, 136, 184304.

${ }^{21}$ M. V Ivanov and D. Babikov, Proc. Natl. Acad. Sci. U. S. A., 2013, 110, $17708-17713$.

${ }^{22}$ M. V. Ivanov and D. Babikov, J. Chem. Phys., 2016, 144, 154301.

${ }^{23}$ A. Teplukhin and D. Babikov, J. Chem. Phys., 2016, under review.

${ }^{24}$ A. Teplukhin and D. Babikov, Chem. Phys. Lett., 2014, 614, 99-103.

${ }^{25}$ G. G. Balint-Kurti and Á. Vibók, in Numerical Grid Methods and Their Application to Schrödinger's Equation, ed. C. Cerjan, Springer Netherlands, Dordrecht, 1993, pp. 195-205.

${ }^{26}$ R. Dawes, P. Lolur, A. Li, B. Jiang and H. Guo, J. Chem. Phys., 2013, 139, 201103.

${ }^{27}$ R. Siebert, R. Schinke and M. Bittererova, Phys. Chem. Chem. Phys., 2001, 3, 1795-1798.

${ }^{28}$ M. Ayouz and D. Babikov, J. Chem. Phys., 2013, 138, 164311.

${ }^{29}$ Y. Li, Z. Sun, B. Jiang, D. Xie, R. Dawes and H. Guo, J. Chem. Phys., 2014, $141,081102$.

30Z. Sun, D. Yu, W. Xie, J. Hou, R. Dawes and H. Guo, J. Chem. Phys., 2015, $142,174312$.

${ }^{31}$ W. Xie, L. Liu, Z. Sun, H. Guo and R. Dawes, J. Chem. Phys., 2015, 142, 064308.

${ }^{32}$ T. R. Rao, G. Guillon, S. Mahapatra and P. Honvault, J. Phys. Chem. Lett., 2015, 6, 633-636.

${ }^{33}$ T. Rajagopala Rao, G. Guillon, S. Mahapatra and P. Honvault, J. Chem. Phys., 2015, 142, 174311.

${ }^{34}$ S. Ndengué, R. Dawes, X.-G. Wang, T. Carrington, Z. Sun and H. Guo, J. Chem. Phys., 2016, 144, 074302.

${ }^{35}$ A. Teplukhin and D. Babikov, J. Chem. Educ., 2015, 92, 305-309.

36Z. Bačić and J. C. Light, J. Chem. Phys., 1986, 85, 4594.

37]. C. Light and T. Carrington, in Adv. Chem. Phys., ed. I. Prigogine and S. A. Rice, John Wiley \& Sons, Inc, Hoboken, NJ, USA, 2000, vol. 114, pp. 263-310.

${ }^{38}$ V. Kokoouline, O. Dulieu, R. Kosloff and F. Masnou-Seeuws, J. Chem. Phys., $1999,110,9865$.

${ }^{39}$ A. G. Borisov, J. Chem. Phys., 2001, 114, 7770-7777.

${ }^{40}$ L. S. Blackford, J. Choi, A. Cleary, E. D’Azeuedo, J. Demmel, I. Dhillon, J. J. Dongarra, S. Hammarling, G. Henry, A. Petitet, K. Stanley, D. Walker and R. C. Whaley, ScaLAPACK user's guide, Society for Industrial and Applied Mathematics, 1997.

${ }^{41}$ R. T. Pack and G. a. Parker, J. Chem. Phys., 1987, 87, 3888.

${ }^{42}$ B. K. Kendrick, R. T. Pack, R. B. Walker and E. F. Hayes, J. Chem. Phys., $1999,110,6673$.

43]. Guenther, D. Krankowsky and K. Mauersberger, Chem. Phys. Lett., 2000, $324,31-36$.

Physical Chemistry Chemical Physics, No. 18 (2016): pg. 19194-19206. DOI. This article is @ Royal Society of Chemistry and permission has been granted for this version to appear in e-Publications@Marquette. Royal Society of Chemistry does not grant permission for this article to be further copied/distributed or hosted elsewhere without the express permission from Royal Society of Chemistry. 
NOT THE PUBLISHED VERSION; this is the author's final, peer-reviewed manuscript. The published version may be accessed by following the link in the citation at the bottom of the page.

${ }^{44}$ H. Hippler, R. Rahn and J. Troe, J. Chem. Phys., 1990, 93, 6560.

${ }^{45} \mathrm{C}$. L. Lin and M. T. Leu, Int. J. Chem. Kinet., 1982, 14, 417-434.

${ }^{46}$ Y. Q. Gao and R. A. Marcus, J. Chem. Phys., 2002, 116, 137-154.

${ }^{47}$ R. T Pack, R. B. Walker and B. K. Kendrick, J. Chem. Phys., 1998, 109, 6714.

${ }^{48}$ M. V. Ivanov, S. Y. Grebenshchikov and R. Schinke, J. Chem. Phys., 2004, 120, 10015-10024.

${ }^{49}$ M. V. Ivanov and R. Schinke, J. Chem. Phys., 2005, 122, 234318.

${ }^{50}$ R. Atkinson, D. L. Baulch, R. A. Cox, R. F. Hampson, J. A. Kerr and J. Troe, J. Phys. Chem. Ref. Data, 1992, 21, 1125.

Physical Chemistry Chemical Physics, No. 18 (2016): pg. 19194-19206. DOI. This article is @ Royal Society of Chemistry and permission has been granted for this version to appear in e-Publications@Marquette. Royal Society of Chemistry does not grant permission for this article to be further copied/distributed or hosted elsewhere without the express permission from Royal Society of Chemistry. 